\title{
Jaynes-Cummings-model with damping at resonance
}

\author{
A. Lindner ${ }^{1, a}$, H. Freese ${ }^{2}$, G. Quehl ${ }^{1}$, D. Rei $\beta^{1}$, and K. Schiller ${ }^{1,3}$ \\ 1 Institut für Laser-Physik der Universität Hamburg, Jungiusstraße 9, 20355 Hamburg, Germany \\ 2 1. Institut für Theoretische Physik der Universität Hamburg, Jungiusstraße 9, 20355 Hamburg, Germany \\ 3 Institut für Physik der Universität Potsdam, Am Neuen Palais 10, 14469 Potsdam, Germany
}

Received 22 January 2001 and Received in final form 16 May 2001

\begin{abstract}
The time evolution of a damped two-level atom coupled to a damped field mode at resonance is calculated analytically as well as numerically in the basis of dressed states. We study the dynamics of the density matrix and of observables, e.g. the number of field quanta. For the initial states we consider the field to be in a Fock or Glauber state and the atom in the ground or excited state. We show the significant influence of the damping on the well-known phenomena of this model, e.g. collapse and revival.
\end{abstract}

PACS. 31.70.Hq Time-dependent phenomena: excitation and relaxation processes, and reaction rates 32.80.-t Photon interactions with atoms - 33.80.-b Photon interactions with molecules - 42.50.Ct Quantum description of interaction of light and matter; related experiments

\section{Introduction}

The Hamiltonian of the Jaynes-Cummings-model [1]

$$
H=\hbar \omega\left(\frac{1}{2} \sigma_{z}+\Psi^{\dagger} \Psi\right)+\frac{1}{2} \hbar g\left(\sigma_{+} \Psi+\sigma_{-} \Psi^{\dagger}\right)
$$

is the commonly preferred framework for describing the interaction of an atom (or ion or molecule) with the electromagnetic field (or the harmonic oscillation of the center of mass of the atom in an external field). The Hamiltonian describes stimulated emission and absorption at resonance - with detuning we would need two frequencies $\omega_{\mathrm{A}} \neq \omega_{\mathrm{L}}$.

In the following we use the eigenstates of the Hamiltonian ("dressed states") [2-4]

$$
|\varepsilon, n\rangle=\frac{|\downarrow, n+1\rangle+\varepsilon|\uparrow, n\rangle}{\sqrt{2}}
$$

with $\varepsilon= \pm$ and $n \in\{0,1, \ldots\}$. In addition we have the ground state $|\downarrow, 0\rangle$ with energy $-\frac{1}{2} \hbar \omega$. This state can formally be included by allowing $n=-1$, as $|\downarrow, 0\rangle=$ $(|+,-1\rangle+|-,-1\rangle) / \sqrt{2}$. The eigenvalues of the Hamiltonian are given by

$$
H|\varepsilon, n\rangle=|\varepsilon, n\rangle \hbar\left\{\left(n+\frac{1}{2}\right) \omega+\varepsilon \frac{1}{2} \Omega_{n}\right\}
$$

\footnotetext{
a e-mail: lindner@physnet.uni-hamburg.de
}

with the Rabi frequency

$$
\Omega_{n}=\sqrt{n+1} g
$$

In order to describe dissipation, e.g. spontaneous emission, one has to treat the system as open. We prefer to use the master equation ("Liouville equation") [5-8]

$$
\begin{aligned}
\frac{\mathrm{d} \varrho}{\mathrm{d} t}=\frac{[H, \varrho]}{\mathrm{i} \hbar} & +\gamma_{\mathrm{A}}\left(\left[\sigma_{-} \varrho, \sigma_{+}\right]+\left[\sigma_{-}, \varrho \sigma_{+}\right]\right) \\
& +\gamma_{\mathrm{L}}\left(\left[\Psi \varrho, \Psi^{\dagger}\right]+\left[\Psi, \varrho \Psi^{\dagger}\right]\right) \\
& +\frac{1}{2} \gamma_{\mathrm{P}}\left(\left[\sigma_{z} \varrho, \sigma_{z}\right]+\left[\sigma_{z}, \varrho \sigma_{z}\right]\right)
\end{aligned}
$$

where the damping of the atom and of the field mode are treated separately, with $\gamma_{\mathrm{A}}$ and $\gamma_{\mathrm{L}}$ being their respective decay constants. A dephasing of the atomic coherence is incorporated by the last term which is usually written as $\gamma_{\mathrm{P}}\left(\sigma_{z} \varrho \sigma_{z}-\varrho\right)$. The environment is assumed to be at zero temperature, hence no thermic excitation is included. The Liouville equation (5) conserves $\operatorname{tr} \varrho=1$ and guarantees that the density operator remains a Hermitian and positive operator as shown by Lindblad [6]. 
We will use the parameters of the microwave experiment at Paris $[9]^{1}$

$$
\begin{aligned}
\omega=321 \mathrm{GHz} \gg & g=151 \mathrm{kHz} \gg \gamma_{\mathrm{L}}=3 \mathrm{kHz} \\
& \approx \gamma_{\mathrm{P}}=2.5 \mathrm{kHz} \gg \gamma_{\mathrm{A}}=17 \mathrm{~Hz} .
\end{aligned}
$$

Because of $\gamma_{\mathrm{L}} \gg \gamma_{\mathrm{A}}$ many authors (e.g. [12-14]) neglect the damping of the atom. However, we will show, that this can straightforwardly be included in the first order as long as the sum $\gamma_{\mathrm{A}}+\gamma_{\mathrm{P}}$ is not significantly larger than $\gamma_{\mathrm{L}}$.

Our method to solve equation (5) in the basis of dressed states (Eq. (2)) is motivated by the physical parameters in equation (6): high quality experiments tend to reduce damping as much as possible. In this strong coupling limit $\left(g \gg \gamma_{\mathrm{L}}, \gamma_{\mathrm{A}}, \gamma_{\mathrm{P}}\right)$ it seems advisable to treat the system in a basis that diagonalizes the Hamiltonian. Only in the opposite regime of strong damping $\left(\gamma_{\mathrm{L}}, \gamma_{\mathrm{A}}, \gamma_{\mathrm{P}} \gg g\right)$ should one use a basis of damped states [15]. In this reference the problem is solved even for detuning $\left(\omega_{\mathrm{A}} \neq \omega_{\mathrm{L}}\right)$ and for $T \neq 0$. This could be achieved, however, only by more elaborate mathematical tools, involving $4 \times 4$-matrix continued fractions and with all the parameters in equations $(1,5)$ whereas we employ an interaction representation with mean damping. In this way the problem needs not to be treated on the very short timescale $1 / \omega$ and all $4 \times 4$-matrices are nearly diagonal: we can approximate the findings by scalar equations and so the problem does not become "rather involved" as suggested in reference [15]. This reference also deals with the number of field quanta through an expression (Eq. (4.16) in their paper) based on an infinite number of "constants $a_{n}, b_{n}, c_{n} \ldots$ which are determined by the initial state of the field". Technically this is correct but there is no further explanation. That is there is no discussion of the initial Glauber state.

In [16] the problem is treated analytically for large detuning.

We are not only interested in the evolution of the density matrix as given by equation (5) or of equivalent quasi probabilities [12-14] but also in the dynamics of observables.

In the next section we express equation (5) as a matrix equation in the basis of dressed states: the damping acts as a non Hermitian "interaction" and we introduce a non unitary transformation to simplify the differential

\footnotetext{
1 In that experiment the "ground state" of our model can decay to a third state, because it is a highly excited Rydberg state. However, there the detector only counts atoms which are either in the ground or in the excited state: atoms in the third (decayed) state do not contribute to the signal. Furthermore the corresponding decay rate is about equal to $\gamma_{\mathrm{A}}$ and thus much smaller than any other relevant frequency. We therefore do not take this effect into account in our model. One might be tempted to also neglect the decay of the excited state to the ground state. We keep this parameter for the generality of the model, which can also be applied to experiments where $\gamma_{\mathrm{A}}$ is considerably larger, e.g. to the experiments at Konstanz (now Garching) [10] $(\omega=2400 \mathrm{THz}, g=101 \mathrm{MHz}$, $\left.\gamma_{\mathrm{L}}=5 \mathrm{MHz}, \gamma_{\mathrm{A}}=9 \mathrm{MHz}\right)$ and Pasadena [11] $(\omega=2210 \mathrm{THz}$, $g=201 \mathrm{MHz}, \gamma_{\mathrm{L}}=13 \mathrm{MHz}, \gamma_{\mathrm{A}}=8 \mathrm{MHz}$ ). (We do not know about the phase decay of these experiments.)
}

equation. This is solved approximately in Section 3 to proceed analytically. The diagonal elements of the density operator (Sect. 4) are especially interesting since they acquire a time dependence through the damping. Here we derive simple expressions for an initial Fock state ("number state") or Glauber state ("coherent state") of the field and ground or excited state of the atom. These matrix elements are used to calculate the number of field quanta in Section 5. In Section 6 we remedy the approximation in Section 3 and describe the correct procedure. However this is only feasible numerically.

After that we show the danger of calculating the time evolution of observables in the Jaynes-Cummings model directly - in the quasi classical approach one neglects the correlation between atom and field and thus collects large errors even without damping (Sect. 7). This problem is circumvented in Section 8 by using a set of four characteristic functions which include all the correlations between field and atom. Their time dependence is given by a system of partial differential equations. In Section 9 this method is demonstrated for an undamped system, where an analytic solution can be found. With damping one has to resort to approximations as shown in Section 10. As an example the time dependence of the number of field quanta is calculated.

\section{Transformation of the Liouville equation}

Starting from equation (5) which can be written as a system of inhomogeneous linear differential equations and with the abbreviation $r_{n}^{ \pm}=\sqrt{n+2} \pm \sqrt{n+1}$ we have

$$
\begin{aligned}
\left\langle\varepsilon, n|\varrho| \varepsilon^{\prime},\right. & \left.n^{\prime}\right\rangle=-\left\langle\varepsilon, n|\varrho| \varepsilon^{\prime}, n^{\prime}\right\rangle\left\{\mathrm{i}\left(n-n^{\prime}\right) \omega\right. \\
& \left.+\frac{\mathrm{i}}{2}\left(\varepsilon \Omega_{n}-\varepsilon^{\prime} \Omega_{n^{\prime}}\right)+\left(n+1+n^{\prime}\right) \gamma_{\mathrm{L}}+\gamma_{\mathrm{A}}+\gamma_{\mathrm{P}}\right\} \\
& -\left(\left\langle\varepsilon, n|\varrho|-\varepsilon^{\prime}, n^{\prime}\right\rangle+\left\langle-\varepsilon, n|\varrho| \varepsilon^{\prime}, n^{\prime}\right\rangle\right) \frac{1}{2}\left(\gamma_{\mathrm{L}}-\gamma_{\mathrm{A}}\right) \\
& +\left\langle-\varepsilon, n|\varrho|-\varepsilon^{\prime}, n^{\prime}\right\rangle \gamma_{\mathrm{P}} \\
& +\left\langle+\varepsilon, n+1|\varrho|+\varepsilon^{\prime}, n^{\prime}+1\right\rangle \frac{1}{2}\left(r_{n}^{+} r_{n^{\prime}}^{+} \gamma_{\mathrm{L}}+\varepsilon \varepsilon^{\prime} \gamma_{\mathrm{A}}\right) \\
& +\left\langle+\varepsilon, n+1|\varrho|-\varepsilon^{\prime}, n^{\prime}+1\right\rangle \frac{1}{2}\left(r_{n}^{+} r_{n^{\prime}}^{-} \gamma_{\mathrm{L}}-\varepsilon \varepsilon^{\prime} \gamma_{\mathrm{A}}\right) \\
& +\left\langle-\varepsilon, n+1|\varrho|+\varepsilon^{\prime}, n^{\prime}+1\right\rangle \frac{1}{2}\left(r_{n}^{-} r_{n^{\prime}}^{+} \gamma_{\mathrm{L}}-\varepsilon \varepsilon^{\prime} \gamma_{\mathrm{A}}\right) \\
& +\left\langle-\varepsilon, n+1|\varrho|-\varepsilon^{\prime}, n^{\prime}+1\right\rangle \frac{1}{2}\left(r_{n}^{-} r_{n^{\prime}}^{-} \gamma_{\mathrm{L}}+\varepsilon \varepsilon^{\prime} \gamma_{\mathrm{A}}\right)
\end{aligned}
$$

The solution of the differential equation (having no homogeneous term)

$$
\begin{array}{r}
\langle\downarrow, 0|\varrho| \downarrow, 0\rangle=(\langle+, 0|\varrho|+, 0\rangle+\langle-, 0|\varrho|-, 0\rangle)\left(\gamma_{\mathrm{L}}+\gamma_{\mathrm{A}}\right) \\
+(\langle+, 0|\varrho|-, 0\rangle+\langle-, 0|\varrho|+, 0\rangle)\left(\gamma_{\mathrm{L}}-\gamma_{\mathrm{A}}\right)
\end{array}
$$

is already given by the other diagonal elements $\langle\varepsilon, n|\varrho| \varepsilon, n\rangle$ because the Liouville equation conserves $\operatorname{tr} \varrho=1$.

Splitting off time dependent factors will help to solve the problem:

$$
\begin{aligned}
\left\langle\varepsilon, n|\varrho| \varepsilon^{\prime}, n^{\prime}\right\rangle= & \exp \left\{-\mathrm{i}\left(n-n^{\prime}\right) \omega t\right\}\left\langle\varepsilon, n|\bar{\varrho}| \varepsilon^{\prime}, n^{\prime}\right\rangle \\
= & \exp \left\{-\left[\mathrm{i}\left(n-n^{\prime}\right) \omega+\left(n+1+n^{\prime}\right) \gamma_{\mathrm{L}}\right.\right. \\
& \left.\left.+\gamma_{\mathrm{A}}+\gamma_{\mathrm{P}}\right] t\right\}\left\langle\varepsilon, n|\widetilde{\varrho}| \varepsilon^{\prime}, n^{\prime}\right\rangle
\end{aligned}
$$


where $\bar{\varrho}$ corresponds to the interaction representation and $\widetilde{\varrho}$ also takes into account the mean damping. Here the atomic energy and phase decay $\gamma_{\mathrm{A}}$ and $\gamma_{\mathrm{P}}$ do not contribute individually but only as a sum. The density matrix for given $n$ and $n^{\prime}$ forms a $2 \times 2$-matrix, which we write as a vector with 4 components:

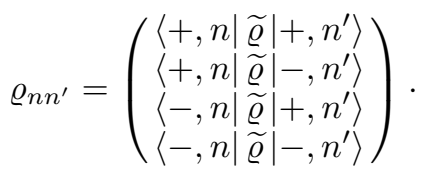

The Liouville equation then appears as

$$
\frac{\mathrm{d} \varrho_{n n^{\prime}}}{\mathrm{d} t}=L_{n n^{\prime}} \varrho_{n n^{\prime}}+\exp \left(-2 \gamma_{\mathrm{L}} t\right) \Gamma_{n n^{\prime}} \varrho_{n+1, n^{\prime}+1} .
$$

Here we used the real $4 \times 4$-matrix

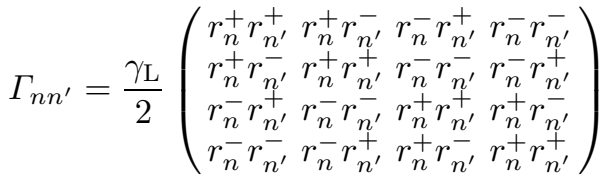

$$
\begin{aligned}
& +\frac{\gamma_{\mathrm{A}}}{2}\left(\begin{array}{llll}
1 & -1 & -1 & 1 \\
1 & -1 & -1 & 1 \\
1 & -1 & -1 & 1 \\
1 & -1 & -1 & 1
\end{array}\right)
\end{aligned}
$$

and the complex and symmetrical

$$
\begin{aligned}
L_{n n^{\prime}}=-\mathrm{i} & \left(\begin{array}{cccc}
\Omega_{n n^{\prime}}^{-} & 0 & 0 & 0 \\
0 & \Omega_{n n^{\prime}}^{+} & 0 & 0 \\
0 & 0 & -\Omega_{n n^{\prime}}^{+} & 0 \\
0 & 0 & 0 & -\Omega_{n n^{\prime}}^{-}
\end{array}\right) \\
& -\frac{\gamma_{\mathrm{L}}-\gamma_{\mathrm{A}}}{2}\left(\begin{array}{cccc}
0 & 1 & 1 & 0 \\
1 & 0 & 0 & 1 \\
1 & 0 & 0 & 1 \\
0 & 1 & 1 & 0
\end{array}\right)+\gamma_{\mathrm{P}}\left(\begin{array}{llll}
0 & 0 & 0 & 1 \\
0 & 0 & 1 & 0 \\
0 & 1 & 0 & 0 \\
1 & 0 & 0 & 0
\end{array}\right)
\end{aligned}
$$

with the frequencies

$$
\Omega_{n n^{\prime}}^{ \pm}=\frac{1}{2}\left(\Omega_{n} \pm \Omega_{n^{\prime}}\right)
$$

(Thus we have $r_{n}^{ \pm}=2 \Omega_{n+1, n}^{ \pm} / g$.)

\section{Solutions of the Liouville equation}

The Liouville equation (12) can be solved after diagonalization of the matrix $L_{n n^{\prime}}$. This transformation is given in Section 6 and was used for a numerical solution. However, because of $\left(\gamma_{\mathrm{L}}, \gamma_{\mathrm{A}}, \gamma_{\mathrm{P}}\right) \ll g$ we neglect the non-diagonal part of $L_{n n^{\prime}}$ (Eq. (14)) for $n \neq n^{\prime}$. In this case the atomic damping is only contained in the time dependent factor previously split off (Eq. (10)). For $n=n^{\prime}$ however, we have $\Omega_{n n^{\prime}}^{-}=0$, so this case has to be considered separately. Furthermore, with regard to $\gamma_{\mathrm{A}} \lesssim \gamma_{\mathrm{L}}$ and $r_{n}^{+} \gg r_{n}^{-}$we can approximate the matrix

$$
\Gamma_{n n^{\prime}} \approx \frac{\gamma_{\mathrm{L}} r_{n}^{+} r_{n^{\prime}}^{+}}{2}\left(\begin{array}{cccc}
1 & 0 & 0 & 0 \\
0 & 1 & 0 & 0 \\
0 & 0 & 1 & 0 \\
0 & 0 & 0 & 1
\end{array}\right) .
$$

With these simplifications the four components of $\varrho_{n n^{\prime}}$ decouple. The differential equation (12) can now be understood as a scalar equation for each component as long as $L_{n n^{\prime}}$ is replaced by the appropriate diagonal element $\mp \mathrm{i} \Omega_{n n^{\prime}}^{\mp}$.

In the case $n=n^{\prime}$ we have $\Omega_{n n^{\prime}}^{-}=0$. Then one has to consider

$$
\varrho_{n n}^{ \pm}(t)=\frac{\langle+, n|\widetilde{\varrho}|+, n\rangle \pm\langle-, n|\widetilde{\varrho}|-, n\rangle}{\sqrt{2}}
$$

with the eigenvalues $L_{n n}= \pm \gamma_{\mathrm{P}}$ instead of the first and fourth elements of the vector $\varrho_{n n}(t)$. The eigenvalues $\pm \gamma_{\mathrm{P}}$ do not depend on $n$ and can obviously be incorporated in equation (10): i.e. $\sum_{\varepsilon}\langle\varepsilon, n|\widetilde{\varrho}| \varepsilon, n\rangle$ does not have the factor $\exp \left(-\gamma_{\mathrm{P}} t\right)$ and the difference $\langle+, n|\widetilde{\varrho}|+, n\rangle-\langle-, n|\widetilde{\varrho}|-, n\rangle$ decays faster than the sum. Thus, in this special case the atomic energy and phase decay $\gamma_{\mathrm{A}}$ and $\gamma_{\mathrm{P}}$ do contribute individually unlike in the previous discussion.

The differential equation can be solved with the ansatz

$$
\varrho_{n n^{\prime}}(t)=\sum_{k=0}^{\infty} a_{n n^{\prime} k} \exp \left[\left(L_{n+k, n^{\prime}+k}-2 k \gamma_{\mathrm{L}}\right) t\right],
$$

if for all pairs $\left(n, n^{\prime}\right)$ not only the initial conditions

$$
\varrho_{n n^{\prime}}(0)=\sum_{k=0}^{\infty} a_{n n^{\prime} k}
$$

but also the equations for all $k \in\{1,2, \ldots\}$

$$
\left(L_{n+k, n^{\prime}+k}-L_{n n^{\prime}}-2 k \gamma_{\mathrm{L}}\right) a_{n n^{\prime} k}=\Gamma_{n n^{\prime}} a_{n+1, n^{\prime}+1, k-1}
$$

are satisfied. If the field excitation is finite, i.e. there exists a maximal quantum number $n_{0}$, the upper limit of the sum is given by $n_{0}-n$.

This is for example the case if the field starts in a Fock state with $n_{\mathrm{i}}$ quanta. If the initial state of the atom is the excited state $|\uparrow\rangle$ (or the ground state $|\downarrow\rangle$ ) we have according to equation (2) $n_{0}=n_{\mathrm{i}}$ (or $n_{0}=n_{\mathrm{i}}-1$ resp.). Therefore all $\varrho_{n n^{\prime}}(0)$ vanish except

$$
\varrho_{n_{0} n_{0}}(0)=\frac{1}{2}\left(\begin{array}{r}
1 \\
\mp 1 \\
\mp 1 \\
1
\end{array}\right),
$$

where the upper (lower) case refers to the atom starting in the excited (ground) state.

For general initial conditions we take advantage of the linearity of the system of differential equations: we decompose $\varrho_{n n^{\prime}}(0)$ in the basis $\varrho_{n_{0} n_{0}{ }^{\prime}}(0)$ and construct the general solution by superposition. Starting with

$$
a_{n_{0} n_{0}{ }^{\prime} k}=\delta_{k 0} \varrho_{n_{0} n_{0}{ }^{\prime}}(0)
$$

and iterating with $n \in\left\{n_{0}-1, \ldots, 0\right\}$

$$
\frac{a_{n-k, n^{\prime}-k, k}}{a_{n n^{\prime} 0}}=\prod_{l=1}^{k} \frac{\Gamma_{n-l, n^{\prime}-l}}{L_{n n^{\prime}}-L_{n-l, n^{\prime}-l}-2 l \gamma_{\mathrm{L}}}
$$


for $k \in\left\{n_{0}-n, \ldots, 1\right\}$, where

$$
a_{n n^{\prime} 0}=-\sum_{k=1}^{n_{0}-n} a_{n n^{\prime} k}
$$

the coefficients $a_{n n^{\prime} k}$ can be evaluated. In equation (23) it is advantageous to approximate $r_{n}^{+} \approx 2 \sqrt{n+\frac{3}{2}}$, i.e.

$$
\Gamma_{n n^{\prime}} \approx 2 \gamma_{\mathrm{L}} \sqrt{n+\frac{3}{2}} \sqrt{n^{\prime}+\frac{3}{2}}
$$

For $n<n_{0}$ the constraint (24) can be incorporated into equation (18), reducing the sum by one term:

$$
\begin{aligned}
& \varrho_{n n^{\prime}}(t)=\exp \left(L_{n n^{\prime}} t\right) \\
& \quad \times \sum_{k=1}^{n_{0}-n} a_{n n^{\prime} k}\left\{\exp \left[\left(L_{n+k, n^{\prime}+k}-L_{n n^{\prime}}-2 k \gamma_{\mathrm{L}}\right) t\right]-1\right\} .
\end{aligned}
$$

For real $\varrho_{n_{0} n_{0}}(0)$ two of these solutions are complex conjugate with respect to the other two.

\section{Diagonal elements of the density matrix}

Without damping $\langle\varepsilon, n|\varrho| \varepsilon, n\rangle$ would be constant in time. With damping however, $\Omega_{n n}^{-}=0=\Omega_{n-l, n-l}^{-}$leads to

$$
a_{n n k}=(-)^{k}\left(\begin{array}{c}
n_{0}+\frac{1}{2} \\
n_{0}-n
\end{array}\right)\left(\begin{array}{c}
n_{0}-n \\
k
\end{array}\right) \varrho_{n_{0} n_{0}}(0),
$$

and thus

$$
\varrho_{n n}(t)=\left(\begin{array}{c}
n_{0}+\frac{1}{2} \\
n_{0}-n
\end{array}\right) \varrho_{n_{0} n_{0}}(0)\left[1-\exp \left(-2 \gamma_{\mathrm{L}} t\right)\right]^{n_{0}-n} .
$$

The binomial coefficient can be expressed by gamma functions or a Pochhammer symbol ([17] Eq. (6.1.22))

$$
(c)_{n}=\frac{\Gamma(c+n)}{\Gamma(c)}=c(c+1) \cdots(c+n-1),
$$

namely:

$$
\left(\begin{array}{c}
n_{0}+\frac{1}{2} \\
k
\end{array}\right)=\frac{\Gamma\left(n_{0}+\frac{3}{2}\right)}{k ! \Gamma\left(n_{0}+\frac{3}{2}-k\right)}=\frac{\left(n_{0}+\frac{3}{2}-k\right)_{k}}{k !} .
$$

Pochhammer symbols will appear on various occasions.

Specializing to an initial Fock state of the field - i.e. starting with $\langle\varepsilon, n|\varrho| \varepsilon, n\rangle=\frac{1}{2} \delta_{n n_{0}}$ (Eq. (21)) - we have

$$
\begin{aligned}
\sum_{\varepsilon}\langle\varepsilon, n|\varrho| \varepsilon, n\rangle & =\exp \left\{-\left[(2 n+1) \gamma_{\mathrm{L}}+\gamma_{\mathrm{A}}\right] t\right\} \\
& \times\left(\begin{array}{c}
n_{0}+\frac{1}{2} \\
n_{0}-n
\end{array}\right)\left[1-\exp \left(-2 \gamma_{\mathrm{L}} t\right)\right]^{n_{0}-n}
\end{aligned}
$$

For the probability of the ground state,

$$
\langle\downarrow, 0|\varrho| \downarrow, 0\rangle=1-\sum_{\varepsilon, n}\langle\varepsilon, n|\varrho| \varepsilon, n\rangle
$$

we use

$$
\left(-n_{0}\right)_{n}=(-)^{n} \frac{\Gamma\left(n_{0}+1\right)}{\Gamma\left(n_{0}-n+1\right)}
$$

and rewrite the binomial coefficient

$$
\left(\begin{array}{c}
n_{0}+\frac{1}{2} \\
n_{0}-n
\end{array}\right)=(-)^{n}\left(\begin{array}{c}
n_{0}+\frac{1}{2} \\
n_{0}
\end{array}\right) \frac{\left(-n_{0}\right)_{n}}{\left(\frac{3}{2}\right)_{n}}
$$

leading us to the hypergeometric function ([17] Eqs. (15.1.1) and (3.4))

$$
\begin{aligned}
F\left(\begin{array}{l|l}
a & b \\
c
\end{array} \mid x\right) & =\sum_{n=0}^{\infty} \frac{(a)_{n}(b)_{n}}{(c)_{n}} \frac{x^{n}}{n !} \\
& =\frac{1}{(1-x)^{a}} F\left(\begin{array}{ll|l}
a & c-b \\
c
\end{array}\right.
\end{aligned}
$$

and the final result

$$
\begin{aligned}
\langle\downarrow, 0|\varrho| \downarrow, 0\rangle= & 1-\exp \left[-\left(\gamma_{\mathrm{L}}+\gamma_{\mathrm{A}}\right) t\right] \\
& \times\left(\begin{array}{c}
n_{0}+\frac{1}{2} \\
n_{0}
\end{array}\right) F\left(\begin{array}{c}
-n_{0} \frac{1}{2} \\
\frac{3}{2}
\end{array} \mid \exp \left(-2 \gamma_{\mathrm{L}} t\right)\right) .
\end{aligned}
$$

Note that ([17] Eq. (15.1.20))

$$
\left(\begin{array}{c}
n_{0}+\frac{1}{2} \\
n_{0}
\end{array}\right)^{-1}=F\left(\begin{array}{cc}
-n_{0} & \frac{1}{2} \\
\frac{3}{2} & 1
\end{array}\right)
$$

canceling the hypergeometric function at $t=0$, hence we get $\langle\downarrow, 0|\varrho| \downarrow, 0\rangle_{t=0}=\delta_{n_{0},-1}$, as expected.

These results are displayed in Figures 1-4. Note that in the case $n=n^{\prime}$ and $\varepsilon=\varepsilon^{\prime}$ (upper part of Fig. 1 and both parts of Fig. 2) the sum and the difference of the matrix elements $\langle+, n|\varrho(t)|+, n\rangle$ and $\langle-, n|\varrho(t)|-, n\rangle$ show different time dependences (as discussed above): the sum does not depend on $\gamma_{\mathrm{P}}$ at all, whereas the difference is additionally damped by $\exp \left(-2 \gamma_{\mathrm{P}} t\right)$. Due to the initial condition in Figure $1\left\langle+, n|\varrho|+, n^{\prime}\right\rangle-\left\langle-, n|\varrho|-, n^{\prime}\right\rangle=0$ the difference remains zero. When starting with a dressed state $\left|\varepsilon, n_{0}\right\rangle$ (Fig. 2) there is a difference depending on $\gamma_{\mathrm{P}}$ : due to the dephasing a population of the complementary states $|-\varepsilon, n\rangle$ builds up.

In all other cases $\left(n \neq n^{\prime}\right.$ or $\left.\varepsilon \neq \varepsilon^{\prime}\right)$ only the sum $\gamma_{\mathrm{A}}+\gamma_{\mathrm{P}}$ is relevant.

If the field starts in a Glauber state, i.e. with $\varrho=$ $|\varepsilon \alpha\rangle\langle\varepsilon \alpha|$, we linearly combine the previous solution (31) according to

$$
\begin{aligned}
& \left\langle\varepsilon, n_{0} \mid \uparrow, \alpha\right\rangle=\frac{\varepsilon}{\sqrt{2}} \exp \frac{-|\alpha|^{2}}{2} \frac{\alpha^{n_{0}}}{\sqrt{n_{0} !}}, \\
& \left\langle\varepsilon, n_{0} \mid \downarrow, \alpha\right\rangle=\frac{1}{\sqrt{2}} \exp \frac{-|\alpha|^{2}}{2} \frac{\alpha^{n_{0}+1}}{\sqrt{\left(n_{0}+1\right) !}} .
\end{aligned}
$$


$\langle\varepsilon, n|\varrho(t)| \varepsilon, n\rangle$ with $n \in\{4,3,2,1,0\}$

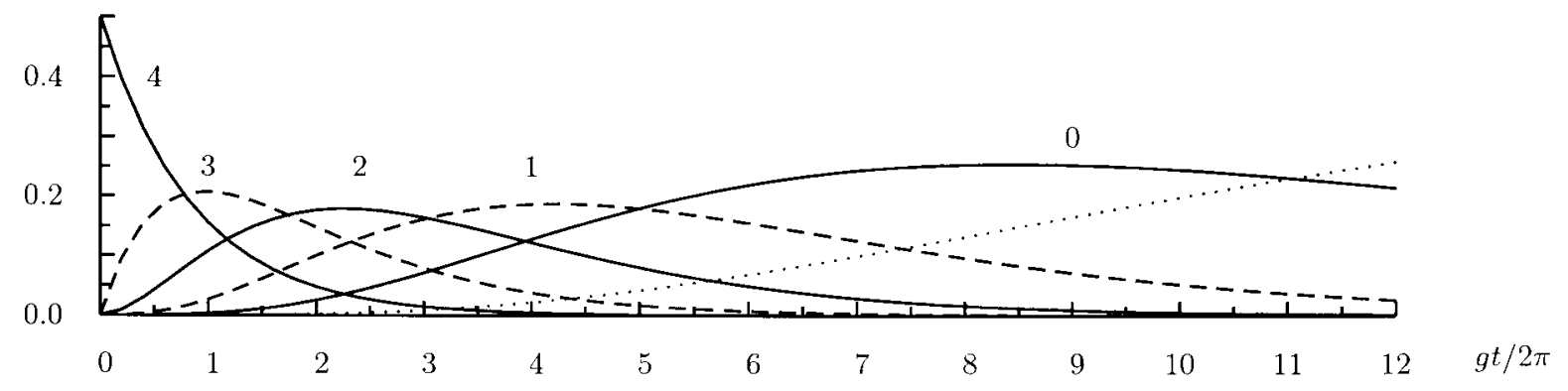

$\operatorname{Re}\langle\varepsilon, n|\varrho(t)|-\varepsilon, n\rangle$ with $n \in\{4,3\}$

$\operatorname{Re}\langle\varepsilon, n|\varrho(t)|-\varepsilon, n\rangle$ with $n \in\{2,1,0\}$
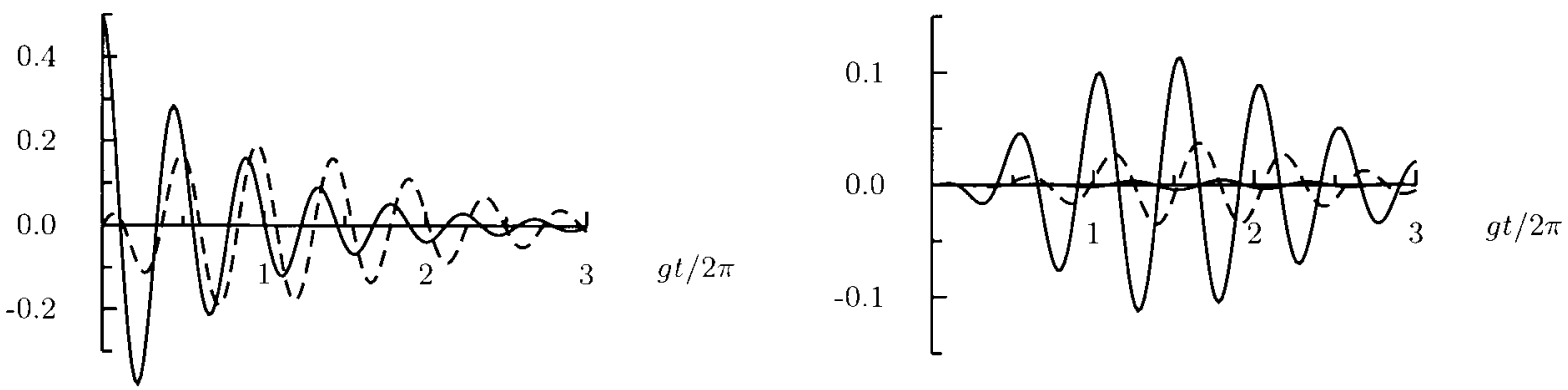

Fig. 1. Density matrix elements $\left\langle\varepsilon, n|\varrho| \varepsilon^{\prime}, n\right\rangle$ : (-) $n$ even, (- - $) n$ odd, $(\cdots) \frac{1}{2}\langle\downarrow, 0|\varrho| \downarrow, 0\rangle$, if initially the atom was in the ground state and there were 5 field quanta. Parameters $\gamma_{\mathrm{L}} / g, \gamma_{\mathrm{P}} / g$ and $\gamma_{\mathrm{A}} / g$ as in equation (6). The upper part is calculated with equation (31) and does not depend on the parameter $\gamma_{\mathrm{P}}$.

$\langle\varepsilon, n|\varrho(t)| \varepsilon, n\rangle$ with $n \in\{4,3,2,1,0\}$

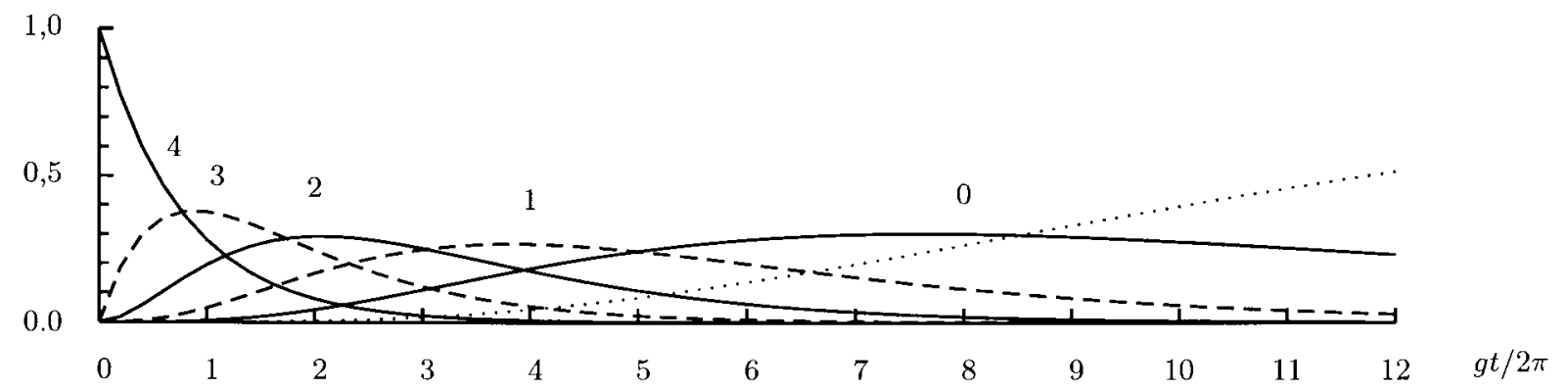

$\langle-\varepsilon, n|\varrho(t)|-\varepsilon, n\rangle$ with $n \in\{4,3,2,1,0\}$

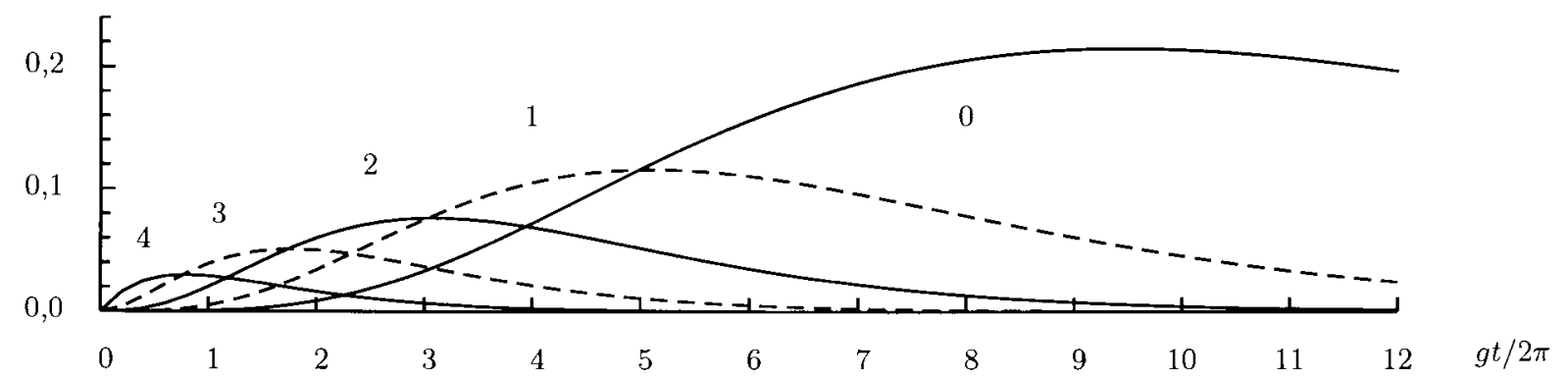

Fig. 2. Diagonal density matrix elements $\langle\varepsilon, n|\varrho(t)| \varepsilon, n\rangle$ in the upper diagram, $\langle-\varepsilon, n|\varrho(t)|-\varepsilon, n\rangle$ in the lower diagram (note the different scaling), when starting purely with the dressed state $\langle\varepsilon, 4|\varrho| \varepsilon, 4\rangle:(-) n$ even, $(---) n$ odd, $(\cdots)\langle\downarrow, 0|\varrho| \downarrow, 0\rangle)$. Parameters as before. The curves (-) and (- - ) follow from equation (31) with an additional factor $\frac{1}{2}\left[1+\exp \left(-2 \gamma_{\mathrm{P}} t\right)\right]($ upper part) and $\frac{1}{2}\left[1-\exp \left(-2 \gamma_{\mathrm{P}} t\right)\right]$ (lower part): with the new initial state the results depend on the parameter $\gamma_{\mathrm{P}}$. 
$\operatorname{Re}\langle\varepsilon, n|\bar{\varrho}| \varepsilon, n-1\rangle$ with $n \in\{4,3,2,1\}$

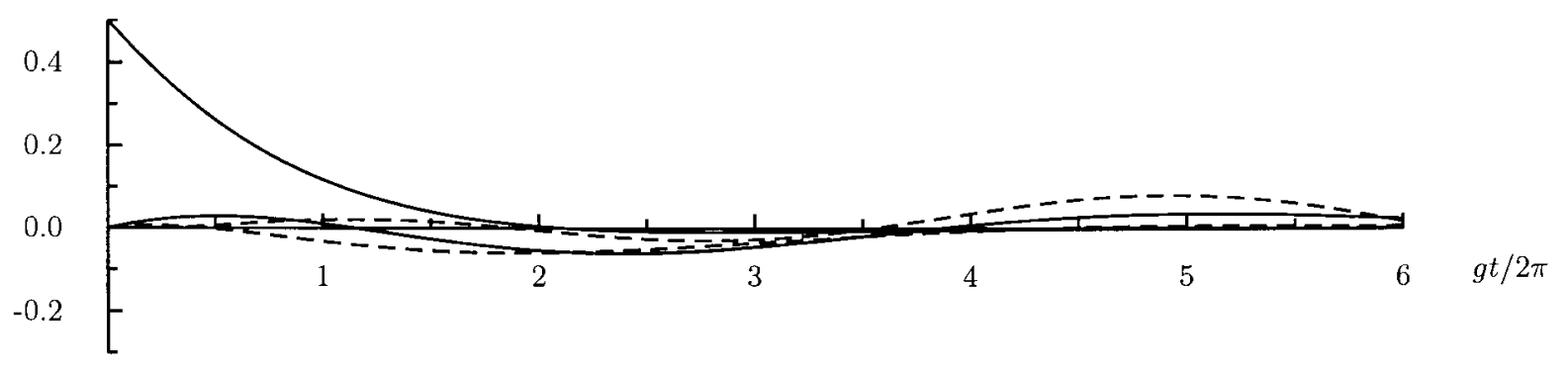

$\operatorname{Re}\langle\varepsilon, n|\underline{\varrho}|-\varepsilon, n-1\rangle$ with $n \in\{4,3\}$

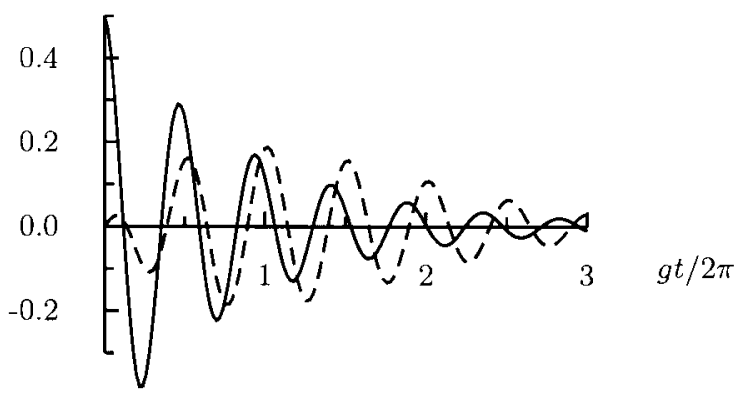

$\operatorname{Re}\langle\varepsilon, n|\bar{\varrho}|-\varepsilon, n-1\rangle$ with $n \in\{2,1\}$

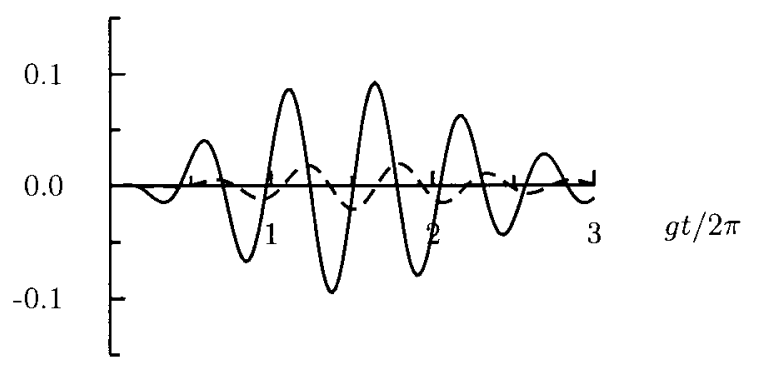

Fig. 3. Density matrix elements $\left\langle\varepsilon, n|\bar{\varrho}| \varepsilon^{\prime}, n-1\right\rangle$ : (-) $n$ even, (- - ) $n$ odd, when starting purely with $\langle \pm, 4|\bar{\varrho}| \pm, 3\rangle=\frac{1}{2}$. Parameters as before. The rather small value of $\operatorname{Re}\left\langle \pm, 3|\varrho| \pm^{\prime}, 2\right\rangle$ is connected with a bigger one of the imaginary part of the matrix element.

$\operatorname{Re}\langle\varepsilon, n|\bar{\varrho}| \varepsilon, n-2\rangle$ with $n \in\{4,3,2\}$

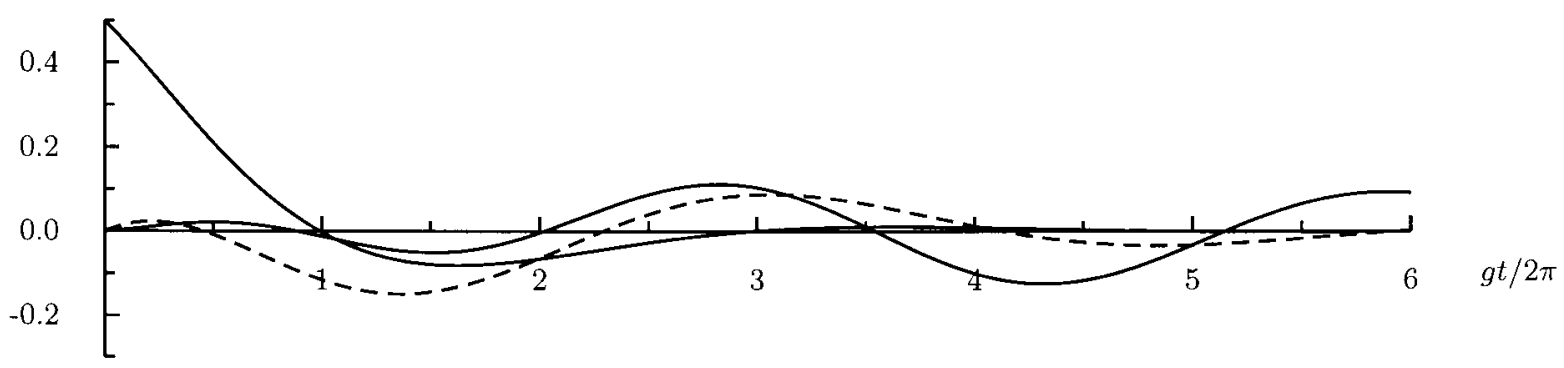

$\operatorname{Re}\langle\varepsilon, n|\bar{\varrho}|-\varepsilon, n-2\rangle$ with $n \in\{4,3\}$

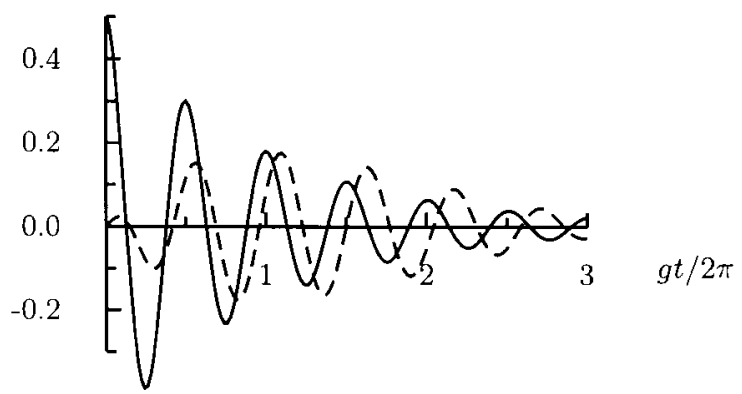

$\operatorname{Re}\langle\varepsilon, n|\bar{\varrho}|-\varepsilon, n-2\rangle$ with $n=2$

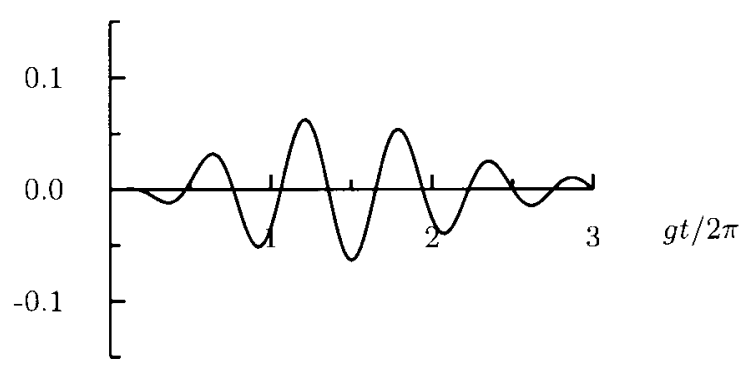

Fig. 4. Density matrix elements $\left\langle\varepsilon, n|\varrho| \varepsilon^{\prime}, n-2\right\rangle$ : (-) $n$ even, (- - ) $n$ odd, when starting purely with $\langle \pm, 4|\varrho| \pm, 2\rangle=\frac{1}{2}$. Parameters as before. 
Since terms with $n_{0}<n$ give no contribution we replace $n_{0}-n \rightarrow m$ and find with the help of equations $(29,30)$ and the confluent hypergeometric function (Kummer's function [17] Eqs. (13.1.2) and (27))

$$
M(a, b, x)=\sum_{m=0}^{\infty} \frac{(a)_{m}}{(b)_{m}} \frac{x^{m}}{m !}=\mathrm{e}^{x} M(b-a, b,-x)
$$

the relation

$$
\begin{aligned}
& \sum_{n_{0}}\left(\begin{array}{l}
n_{0}+\frac{1}{2} \\
n_{0}-n
\end{array}\right) \frac{\left(|\alpha|^{2}-\tau\right)^{n_{0}-n}}{\left(n_{0}+\frac{1}{2} \mp \frac{1}{2}\right) !}= \\
& \frac{M\left(n+\frac{3}{2}, n+\frac{3}{2} \mp \frac{1}{2},|\alpha|^{2}-\tau\right)}{\Gamma\left(n+\frac{3}{2} \mp \frac{1}{2}\right)} .
\end{aligned}
$$

Here we have used the abbreviation

$$
\tau=|\alpha|^{2} \exp \left(-2 \gamma_{\mathrm{L}} t\right)
$$

and finally find

$$
\begin{aligned}
\sum_{\varepsilon}\langle\varepsilon, n|\varrho| \varepsilon, n\rangle= & \exp \left[-\left(\gamma_{\mathrm{L}}+\gamma_{\mathrm{A}}\right) t-\tau\right] \frac{\tau^{n}}{n !} \\
& \times\left\{\begin{array}{l}
M\left(\frac{1}{2}, n+1, \tau-|\alpha|^{2}\right), \\
\frac{|\alpha|^{2}}{n+1} M\left(\frac{1}{2}, n+2, \tau-|\alpha|^{2}\right),
\end{array}\right.
\end{aligned}
$$

according to the initial state of the atom: the upper case corresponds to the excited atom, the lower one to the ground state.

\section{Expectation values: the number of field quanta}

Let us evaluate, how the number of field quanta depends on time in the presence of damping. Equation (2) results in

$$
\left\langle\Psi^{\dagger} \Psi\right\rangle=\sum_{\varepsilon, n}\left(n+\frac{1}{2}\right)\langle\varepsilon, n|\varrho| \varepsilon, n\rangle+\frac{1}{2}\langle\varepsilon, n|\varrho|-\varepsilon, n\rangle .
$$

If there was initially a Fock state with $n_{\mathrm{i}}$ quanta, we find with equation (31)

$$
\sum_{\varepsilon, n}\left(n+\frac{1}{2}\right)\langle\varepsilon, n|\varrho| \varepsilon, n\rangle=\left(n_{\mathrm{i}} \pm \frac{1}{2}\right) \exp \left[-\left(2 \gamma_{\mathrm{L}}+\gamma_{\mathrm{A}}\right) t\right],
$$

since

$$
\sum_{n}\left(n+\frac{1}{2}\right)\left(\begin{array}{l}
n_{0}+\frac{1}{2} \\
n_{0}-n
\end{array}\right) z^{n}=\left(n_{0}+\frac{1}{2}\right) \sqrt{z}(1+z)^{n_{0}-1 / 2} .
$$

Furthermore we have in this case

$$
\frac{1}{2} \sum_{\varepsilon, n}\langle\varepsilon, n|\varrho|-\varepsilon, n\rangle=\mp \frac{1}{2} \sum_{n}\langle+, n|\varrho|-, n\rangle,
$$

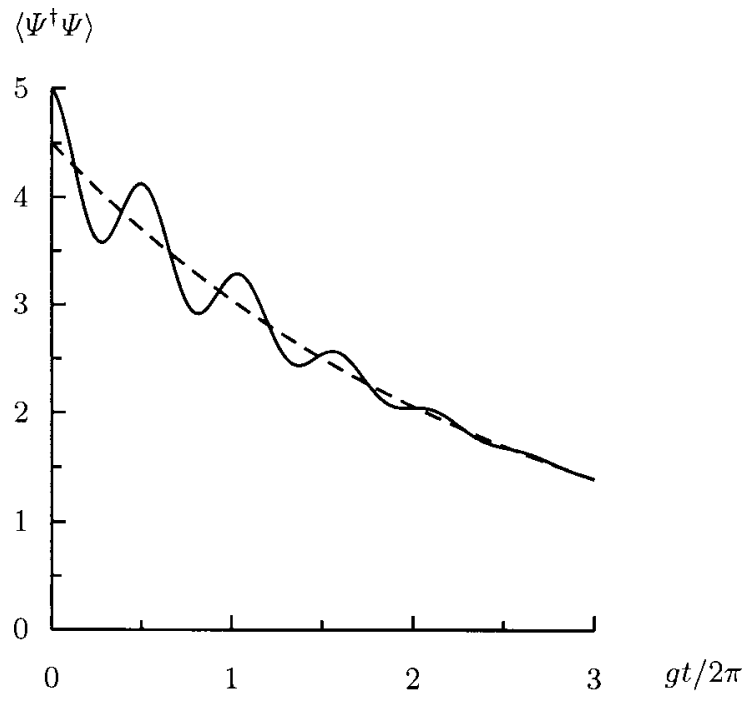

Fig. 5. The number of field quanta, if one starts in a Fock state with 5 photons and the atom in the ground state, remaining parameters as before (-). The curve (- - ) displays the main contribution $\sum_{\varepsilon, n}\left(n+\frac{1}{2}\right)\langle\varepsilon, n|\varrho| \varepsilon, n\rangle$ given by equation (45).

but this sum can only be evaluated numerically. An example is shown in Figure 5.

If the field started in the Glauber state $|\alpha\rangle$, equation (43) yields

$$
\begin{aligned}
& \sum_{\varepsilon, n}\left(n+\frac{1}{2}\right)\langle\varepsilon, n|\varrho| \varepsilon, n\rangle=|\alpha|^{1 \mp 1} \exp \left[-\left(\gamma_{\mathrm{L}}+\gamma_{\mathrm{A}}\right) t-\tau\right] \\
& \quad \times \sum_{n} \frac{\left(n+\frac{1}{2}\right) \tau^{n}}{\Gamma\left(n+\frac{3}{2} \mp \frac{1}{2}\right)} M\left(\frac{1}{2}, n+\frac{3}{2} \mp \frac{1}{2}, \tau-|\alpha|^{2}\right) .
\end{aligned}
$$

This expression can be simplified considerably as shown in Figure 6 and explained subsequently.

By expanding in powers of $\tau-|\alpha|^{2}$ instead of $\tau$, i.e. by using the series in equation (40), and utilizing

$$
\begin{aligned}
\Gamma\left(n+\frac{3}{2} \mp \frac{1}{2}\right)\left(n+\frac{3}{2} \mp \frac{1}{2}\right)_{m} & =\Gamma\left(m+n+\frac{3}{2} \mp \frac{1}{2}\right) \\
& =\Gamma\left(m+\frac{3}{2} \mp \frac{1}{2}\right)\left(m+\frac{3}{2} \mp \frac{1}{2}\right)_{n}
\end{aligned}
$$

we get

$$
\begin{aligned}
& \sum_{n} \frac{\left(n+\frac{1}{2}\right) \tau^{n}}{\left(m+\frac{3}{2} \mp \frac{1}{2}\right)_{n}}=\left(\tau \frac{\partial}{\partial \tau}+\frac{1}{2}\right) M\left(1, m+\frac{3}{2} \mp \frac{1}{2}, \tau\right) \\
& \quad=\tau \frac{M\left(2, m+\frac{5}{2} \mp \frac{1}{2}, \tau\right)}{m+\frac{3}{2} \mp \frac{1}{2}}+\frac{M\left(1, m+\frac{3}{2} \mp \frac{1}{2}, \tau\right)}{2} .
\end{aligned}
$$

Taking the asymptotic values $\tau \gg 1$ of Kummer's functions ([17] Eq. (13.5.1)) this expression can be transformed into the product $\Gamma\left(m+\frac{3}{2} \mp \frac{1}{2}\right) \mathrm{e}^{\tau}\left(\tau \pm \frac{1}{2}-m\right) / \tau^{m+(1 \mp 1) / 2}$ and we find

$$
\begin{aligned}
\sum_{\varepsilon, n}(n+ & \left.\frac{1}{2}\right)\langle\varepsilon, n|\varrho| \varepsilon, n\rangle=\exp \left[-\left(\gamma_{\mathrm{A}} \pm \gamma_{\mathrm{L}}\right) t\right] \\
& \times \sum_{m}\left(\tau \pm \frac{1}{2}-m\right)\left(\frac{1}{2}\right)_{m} \frac{\left[1-\exp \left(2 \gamma_{\mathrm{L}} t\right)\right]^{m}}{m !}
\end{aligned}
$$




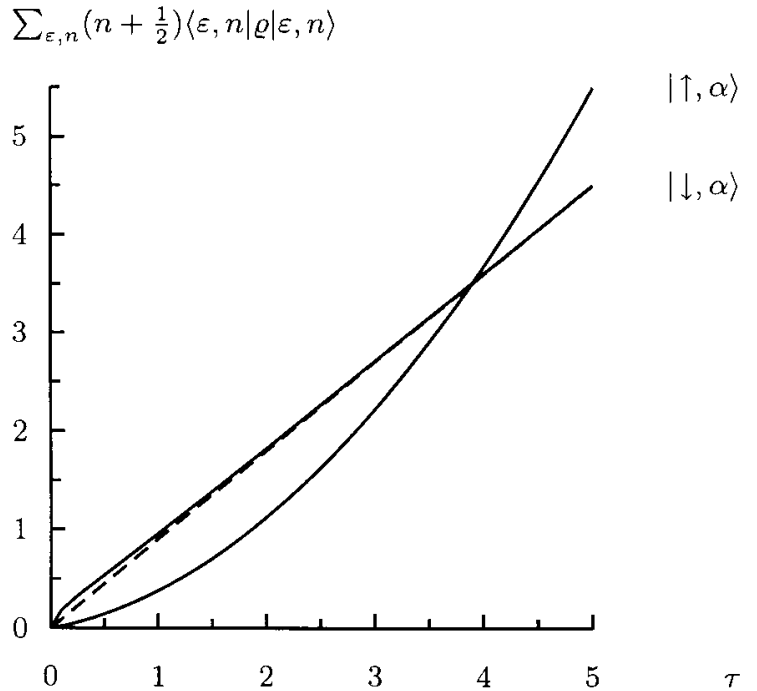

Fig. 6. Comparison of the expressions in equation (47) (-) and equation (51) (- - ) for $|\alpha|^{2}=5$ after splitting off the factor $\exp \left\{-\left(\gamma_{\mathrm{A}}+\gamma_{\mathrm{P}}\right) t\right\}$ : there is no noticeable difference if the atom was initially excited $(|\uparrow, \alpha\rangle)$, and only a small deviation if it began in the ground state $(|\downarrow, \alpha\rangle)$. (Of course the approximation Eq. (51) can only fail for small $\tau \equiv|\alpha|^{2} \exp \left(-2 \gamma_{\mathrm{L}} t\right)$, i.e. large $t$.)

We have ([17] Eq. (15.1.8)) $\sum_{m}(a)_{m} x^{m} / m !=(1-x)^{-a}$ and in addition $\left(\frac{1}{2}\right)_{m+1}=\frac{1}{2}\left(\frac{3}{2}\right)_{m}$. Thus equation (47) can be simplified to

$$
\begin{aligned}
\sum_{\varepsilon, n}\left(n+\frac{1}{2}\right)\langle\varepsilon, n|\varrho| \varepsilon, n\rangle=\exp \left[-\left(2 \gamma_{\mathrm{L}}+\gamma_{\mathrm{A}}\right) t\right] \\
\times\left\{\begin{array}{l}
\left(|\alpha|^{2}-\frac{1}{2}\right) \exp \left(-2 \gamma_{\mathrm{L}} t\right)+1 \\
|\alpha|^{2}-\frac{1}{2}
\end{array}\right.
\end{aligned}
$$

If the atom was initially in the ground state (lower case), this result for a field of an initial Glauber state $|\alpha\rangle$ resembles that of a field of an initial Fock state (Eq. (45)) with $n_{\mathrm{i}}=|\alpha|^{2}$.

\section{Diagonalization of the Liouville equation}

The Liouville equation (12) can be solved exactly after diagonalization of the matrix $L_{n n^{\prime}}$. We call this diagonal matrix $O_{n n^{\prime}}=T_{n n^{\prime}} L_{n n^{\prime}} T_{n n^{\prime}}-1$ and use the vector

$$
P_{n n^{\prime}}(t)=T_{n n^{\prime}} \varrho_{n n^{\prime}}(t)
$$

to get

$$
\begin{aligned}
\dot{P}_{n n^{\prime}} & =O_{n n^{\prime}} P_{n n^{\prime}} \\
& +\exp \left(-2 \gamma_{\mathrm{L}} t\right) T_{n n^{\prime}} \Gamma_{n n^{\prime}} T_{n+1, n^{\prime}+1}{ }^{-1} P_{n+1, n^{\prime}+1} .
\end{aligned}
$$

The eigenvalues follow from an equation of fourth degree. In the cases of biquadratic characteristic equations, namely if $\gamma_{\mathrm{P}}=0$ or if $\gamma_{\mathrm{L}}=\gamma_{\mathrm{A}}$, the solutions can be given in a simple analytical form.

\subsection{Case $\gamma_{P}=0$}

In order to write the transformation matrix $T_{n n^{\prime}}$ in a simple form we choose

$$
\omega_{n}=\sqrt{\Omega_{n}^{2}-\left(\gamma_{\mathrm{L}}-\gamma_{\mathrm{A}}\right)^{2}}
$$

and

$$
a_{n}=\frac{\gamma_{\mathrm{L}}-\gamma_{\mathrm{A}}}{\omega_{n}+\Omega_{n}}
$$

which gives

$$
\begin{aligned}
T_{n n^{\prime}}=\frac{1}{\sqrt{1-a_{n}^{2}} \sqrt{1-a_{n^{\prime}}}} & \\
& \times\left(\begin{array}{cccc}
1 & \mathrm{i} a_{n^{\prime}} & -\mathrm{i} a_{n} & a_{n} a_{n^{\prime}} \\
\mathrm{i} a_{n^{\prime}} & -1 & a_{n} a_{n^{\prime}} & \mathrm{i} a_{n} \\
-\mathrm{i} a_{n} & a_{n} a_{n^{\prime}} & -1 & -\mathrm{i} a_{n^{\prime}} \\
a_{n} a_{n^{\prime}} & \mathrm{i} a_{n} & -\mathrm{i} a_{n^{\prime}} & 1
\end{array}\right) .
\end{aligned}
$$

Note that in this form $T_{n n^{\prime}}=T_{n n^{\prime}}{ }^{-1}$ so that $\varrho_{n n^{\prime}}=$ $T_{n n^{\prime}} P_{n n^{\prime}}$. Now, using the frequencies

$$
\omega_{n n^{\prime}}^{ \pm}=\frac{1}{2}\left(\omega_{n} \pm \omega_{n^{\prime}}\right)
$$

we find

$$
O_{n n^{\prime}}=-\mathrm{i}\left(\begin{array}{cccc}
\omega_{n n^{\prime}}^{-} & 0 & 0 & 0 \\
0 & \omega_{n n^{\prime}}^{+} & 0 & 0 \\
0 & 0 & -\omega_{n n^{\prime}}^{+} & 0 \\
0 & 0 & 0 & -\omega_{n n^{\prime}}^{-}
\end{array}\right)
$$

Since the damping is assumed to be small we have $\omega_{n} \approx$ $\Omega_{n}, \omega_{n n^{\prime}}^{ \pm} \approx \Omega_{n n^{\prime}}^{ \pm}$and $a_{n} \ll 1$. This renders our procedure described in the previous sections to be a good approximation.

\subsection{Case $\gamma_{\mathrm{L}}=\gamma_{\mathrm{A}}$}

In the case $\gamma_{\mathrm{L}}=\gamma_{\mathrm{A}}$ the biquadratic characteristic equation yields the two eigenvalues $+\mathrm{i} \sqrt{\Omega_{n n^{\prime}}^{ \pm}-\gamma_{\mathrm{P}}^{2}}$ and two with the opposite sign. This simplifies to $\mathrm{i} \Omega_{n n^{\prime}}^{ \pm}$for $\gamma_{\mathrm{P}} \ll g$, but for $n=n^{\prime}$ the root with the lower sign is equal to $\mathrm{i} \gamma_{\mathrm{P}}$ : thus the already mentioned eigenvalues $\pm \gamma_{\mathrm{P}}$ replace the vanishing eigenvalues $\pm \mathrm{i} \Omega_{n n}^{-}$.

\section{Time dependence of expectation values}

In principle it is sufficient to know the time dependence of the density matrix. Yet, one then needs the full initial state, i.e. all density matrix elements $\left\langle \pm, n|\varrho| \pm, n^{\prime}\right\rangle$ and $\left\langle \pm, n|\varrho| \mp, n^{\prime}\right\rangle$ with $n, n^{\prime} \in\{0,1, \ldots\}$ at time 0 .

On the other hand we are normally interested only in a few observables, e.g. $\left\langle\Psi^{\dagger} \Psi\right\rangle,\langle\Psi\rangle$ and $\left\langle\sigma_{i}\right\rangle$. Therefore we do not need to know all elements of the density matrix. It appears tempting to concentrate on the relevant observables and to find their time dependence via

$$
\frac{\mathrm{d}\langle A\rangle}{\mathrm{d} t}=\operatorname{tr}\left(A \frac{\mathrm{d} \varrho}{\mathrm{d} t}\right) \text {. }
$$



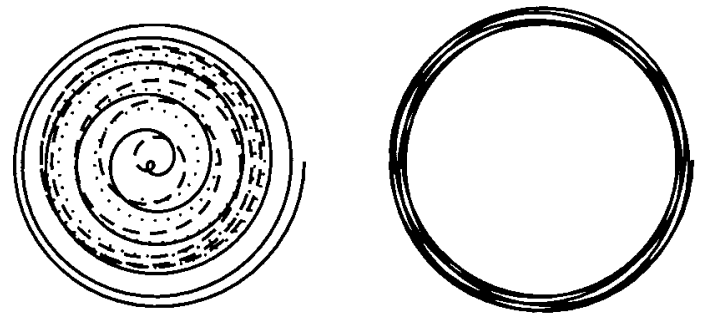

Fig. 7. $\left\langle P / p_{0}\right\rangle$ as a function of $\left\langle X / x_{0}\right\rangle$ in the course of time (damping neglected and after separation of the trivial time dependence $\exp (-\mathrm{i} \omega t))$ - left figure with correlations, right without correlations. In the left figure the solid line develops into the broken one and afterwards into the dotted one: the correlations produce the well-known collapse [24] and revival [25] of the initial state. Parameters: initially the atom was in the ground state and there were 5 photons in a Glauber state $(\alpha=\sqrt{5})$.

But in our case of a coupling between atom and field and damping processes we find

$$
\begin{aligned}
\frac{\mathrm{d}\langle\Psi\rangle}{\mathrm{d} t} & =-\left(\mathrm{i} \omega+\gamma_{\mathrm{L}}\right)\langle\Psi\rangle-\frac{\mathrm{i}}{2} g\left\langle\sigma_{-}\right\rangle, \\
\frac{\mathrm{d}\left\langle\sigma_{-}\right\rangle}{\mathrm{d} t} & =-\left(\mathrm{i} \omega+\gamma_{\mathrm{A}}+2 \gamma_{\mathrm{P}}\right)\left\langle\sigma_{-}\right\rangle+\frac{\mathrm{i}}{2} g\left\langle\Psi \sigma_{z}\right\rangle, \\
\frac{\mathrm{d}\left\langle\sigma_{z}\right\rangle}{\mathrm{d} t} & =-2 \gamma_{\mathrm{A}}\left\langle 1+\sigma_{z}\right\rangle+2 g \operatorname{Im}\left\langle\Psi \sigma_{+}\right\rangle, \\
\frac{\mathrm{d}\left\langle\Psi^{\dagger} \Psi\right\rangle}{\mathrm{d} t} & =-2 \gamma_{\mathrm{L}}\left\langle\Psi^{\dagger} \Psi\right\rangle-g \operatorname{Im}\left\langle\Psi \sigma_{+}\right\rangle,
\end{aligned}
$$

and so on: there is a coupling to more and more observables - with higher and higher powers of $\Psi$ and $\Psi^{\dagger}$.

It is alluring to break off this system of equations by neglecting higher correlations of $\left\langle f\left(\Psi, \Psi^{\dagger}\right) \sigma_{i}\right\rangle$ and to factorize this expression by $\left\langle f\left(\Psi, \Psi^{\dagger}\right)\right\rangle\left\langle\sigma_{i}\right\rangle$ : this is used e.g. in the well known quasi classical approximation - where atoms are described quantum mechanically, but the electromagnetic field is described classically. However, this procedure is faulty, as shown on various occasions [18-23] and can be seen from Figure 7 .

\section{Characteristic functions}

The appearance of more and more expectation values can be circumvented with the help of the (normally ordered) characteristic function

$$
C\left(\xi, \xi^{*}\right)=\langle F\rangle
$$

with

$$
F=\exp \left(\xi \Psi^{\dagger}\right) \exp \left(-\xi^{*} \Psi\right)
$$

This function of the two parameters $\xi$ and $\xi^{*}$ allows the derivation of all expectation values of $\Psi^{\dagger m} \Psi^{n}$ using

$$
\left\langle\Psi^{\dagger} \Psi^{n}\right\rangle=\left.(-)^{n} \frac{\partial^{m+n} C}{\partial \xi^{m} \partial \xi^{* n}}\right|_{\xi=\xi^{*}=0} .
$$

Due to

$$
[F, \Psi]=-\xi F, \quad\left[F, \Psi^{\dagger}\right]=-\xi^{*} F
$$

and

$$
F \Psi=-\frac{\partial F}{\partial \xi^{*}}, \quad \Psi^{\dagger} F=\frac{\partial F}{\partial \xi}
$$

equation (12) leads to the system of partial differential equations

$$
\begin{aligned}
\frac{\partial}{\partial t}\langle F\rangle= & -\left\{\left(\gamma_{\mathrm{L}}-\mathrm{i} \omega\right) \xi \frac{\partial}{\partial \xi}+\left(\gamma_{\mathrm{L}}+\mathrm{i} \omega\right) \xi^{*} \frac{\partial}{\partial \xi^{*}}\right\}\langle F\rangle \\
& +\frac{\mathrm{i}}{2} g \xi\left\langle F \sigma_{+}\right\rangle+\frac{\mathrm{i}}{2} g \xi^{*}\left\langle F \sigma_{-}\right\rangle, \\
\frac{\partial}{\partial t}\left\langle F \sigma_{z}\right\rangle= & -\left\{\left(\gamma_{\mathrm{L}}-\mathrm{i} \omega\right) \xi \frac{\partial}{\partial \xi}+\left(\gamma_{\mathrm{L}}+\mathrm{i} \omega\right) \xi^{*} \frac{\partial}{\partial \xi^{*}}\right\}\left\langle F \sigma_{z}\right\rangle \\
& -2 \gamma_{\mathrm{A}}\left\langle F+F \sigma_{z}\right\rangle \\
& +\mathrm{i} g\left(\frac{\partial}{\partial \xi^{*}}-\frac{1}{2} \xi\right)\left\langle F \sigma_{+}\right\rangle+\mathrm{i} g\left(\frac{\partial}{\partial \xi}-\frac{1}{2} \xi^{*}\right)\left\langle F \sigma_{-}\right\rangle, \\
\frac{\partial}{\partial t}\left\langle F \sigma_{+}\right\rangle= & -\left\{\left(\gamma_{\mathrm{L}}-\mathrm{i} \omega\right) \xi \frac{\partial}{\partial \xi}+\left(\gamma_{\mathrm{L}}+\mathrm{i} \omega\right) \xi^{*} \frac{\partial}{\partial \xi^{*}}\right\}\left\langle F \sigma_{+}\right\rangle \\
& -\left(\gamma_{\mathrm{A}}+2 \gamma_{\mathrm{P}}-\mathrm{i} \omega\right)\left\langle F \sigma_{+}\right\rangle \\
& +\frac{\mathrm{i}}{4} g \xi^{*}\langle F\rangle-\frac{\mathrm{i}}{2} g\left(\frac{\partial}{\partial \xi}-\frac{1}{2} \xi^{*}\right)\left\langle F \sigma_{z}\right\rangle, \\
\frac{\partial}{\partial t}\left\langle F \sigma_{-}\right\rangle= & -\left\{\left(\gamma_{\mathrm{L}}-\mathrm{i} \omega\right) \xi \frac{\partial}{\partial \xi}+\left(\gamma_{\mathrm{L}}+\mathrm{i} \omega\right) \xi^{*} \frac{\partial}{\partial \xi^{*}}\right\}\left\langle F \sigma_{-}\right\rangle \\
& -\left(\gamma_{\mathrm{A}}+2 \gamma_{\mathrm{P}}+\mathrm{i} \omega\right)\left\langle F \sigma_{-}\right\rangle \\
& +\frac{\mathrm{i}}{4} g \xi\langle F\rangle-\frac{\mathrm{i}}{2} g\left(\frac{\partial}{\partial \xi^{*}}-\frac{1}{2} \xi\right)\left\langle F \sigma_{z}\right\rangle .
\end{aligned}
$$

This can be simplified by the transformations

$$
C_{0}=\left\langle F+F \sigma_{z}\right\rangle, \quad C_{1}=\left\langle F-F \sigma_{z}\right\rangle
$$

and

$$
C_{ \pm}=\mathrm{ie}^{\mp \mathrm{i} \omega t}\left\langle F \sigma_{ \pm}\right\rangle
$$

and by the transition to rotating coordinates

$$
\zeta=\xi \mathrm{e}^{+\mathrm{i} \omega t}, \quad \zeta^{*}=\xi^{*} \mathrm{e}^{-\mathrm{i} \omega t},
$$

resulting in

$$
\begin{aligned}
\frac{\partial}{\partial t} C_{0}= & -\gamma_{\mathrm{L}}\left(\zeta \frac{\partial}{\partial \zeta}+\zeta^{*} \frac{\partial}{\partial \zeta^{*}}\right) C_{0}-2 \gamma_{\mathrm{A}} C_{0} \\
& +g\left(\frac{\partial}{\partial \zeta^{*}} C_{+}+\frac{\partial}{\partial \zeta} C_{-}\right) \\
\frac{\partial}{\partial t} C_{1}= & -\gamma_{\mathrm{L}}\left(\zeta \frac{\partial}{\partial \zeta}+\zeta^{*} \frac{\partial}{\partial \zeta^{*}}\right) C_{1}+2 \gamma_{\mathrm{A}} C_{0} \\
& -g\left(\frac{\partial}{\partial \zeta^{*}} C_{+}+\frac{\partial}{\partial \zeta} C_{-}-\zeta C_{+}-\zeta^{*} C_{-}\right), \\
\frac{\partial}{\partial t} C_{+}= & -\gamma_{\mathrm{L}}\left(\zeta \frac{\partial}{\partial \zeta}+\zeta^{*} \frac{\partial}{\partial \zeta^{*}}\right) C_{+}-\left(\gamma_{\mathrm{A}}+2 \gamma_{\mathrm{P}}\right) C_{+} \\
& +\frac{1}{4} g \frac{\partial}{\partial \zeta}\left(C_{0}-C_{1}\right)-\frac{1}{4} g \zeta^{*} C_{0}, \\
\frac{\partial}{\partial t} C_{-}= & -\gamma_{\mathrm{L}}\left(\zeta \frac{\partial}{\partial \zeta}+\zeta^{*} \frac{\partial}{\partial \zeta^{*}}\right) C_{-}-\left(\gamma_{\mathrm{A}}+2 \gamma_{\mathrm{P}}\right) C_{-} \\
& +\frac{1}{4} g \frac{\partial}{\partial \zeta^{*}}\left(C_{0}-C_{1}\right)-\frac{1}{4} g \zeta C_{0} .
\end{aligned}
$$

This system of partial differential equations can be transformed to a system of ordinary differential equations by separation: we split off common factors $\zeta^{m}$ or $\zeta^{* m}$ with $m \in\{1,2, \ldots\}$ and use variables

$$
x=\zeta^{*} \zeta
$$

and $t$. The dependence on $x$ requires special functions, which will be presented in the following sections. 


\section{Undamped solutions}

In order to solve the coupled system (76-79) we will at first concentrate on the main contributions $(\propto g)$ and neglect the damping. The solution in this case introduces the notations needed in Section 10 to describe damping.

With $\gamma_{\mathrm{L}}=\gamma_{\mathrm{A}}=\gamma_{\mathrm{P}}=0$ the system simplifies considerably. In this case the eigenfrequencies are $\Omega_{n n^{\prime}}^{ \pm}$which now (for $n \neq n^{\prime}$ ) will be written as $\Omega_{n+m, n}^{ \pm}$. We employ the pair of auxiliary functions

$$
\begin{aligned}
a_{n m}^{ \pm}(t)= & a_{n m}^{ \pm}(0) \cos \left(\Omega_{n+m, n}^{ \pm} t\right) \\
& +\bar{a}_{n m}^{ \pm}(0) \sin \left(\Omega_{n+m, n}^{ \pm} t\right), \\
\bar{a}_{n m}^{ \pm}(t)= & \bar{a}_{n m}^{ \pm}(0) \cos \left(\Omega_{n+m, n}^{ \pm} t\right) \\
& -a_{n m}^{ \pm}(0) \sin \left(\Omega_{n+m, n}^{ \pm} t\right),
\end{aligned}
$$

and a second pair $\left(b_{n m}^{ \pm}(t), \bar{b}_{n m}^{ \pm}(t)\right)$ of the same form. For $n=n^{\prime}$ (i.e. $m=0$ ) we have $\Omega_{n n}^{+}=\Omega_{n}$,

$$
\begin{aligned}
& a_{n 0}^{+}(t)=a_{n 0}^{+}(0) \cos \left(\Omega_{n} t\right)+\bar{a}_{n 0}^{+}(0) \sin \left(\Omega_{n} t\right), \\
& \bar{a}_{n 0}^{+}(t)=\bar{a}_{n 0}^{+}(0) \cos \left(\Omega_{n} t\right)-a_{n 0}^{+}(0) \sin \left(\Omega_{n} t\right),
\end{aligned}
$$

while $\Omega_{n n}^{-}=0$ leads to constant $a_{n 0}^{-}$and $\bar{a}_{n 0}^{-}$. The contribution of the ground state to the frequency spectrum appears as $\frac{1}{2} \Omega_{n}\left(=\Omega_{n,-1}^{ \pm}\right)$leading to

$$
\begin{aligned}
A_{n}(t)= & A_{n}(0) \cos \left(\frac{1}{2} \Omega_{n} t\right) \\
& +\frac{g}{\frac{1}{2} \Omega_{n}} \bar{A}_{n}(0) \sin \left(\frac{1}{2} \Omega_{n} t\right), \\
\bar{A}_{n}(t)= & \bar{A}_{n}(0) \cos \left(\frac{1}{2} \Omega_{n} t\right) \\
& -\frac{\frac{1}{2} \Omega_{n}}{g} A_{n}(0) \sin \left(\frac{1}{2} \Omega_{n} t\right),
\end{aligned}
$$

and $B_{n}(t)$ and $\bar{B}_{n}(t)$ of the same form, and to a constant $A$ because of $\Omega_{-1,-1}^{ \pm}=0$.

With these abbreviations the solutions can be written as - with $m \in\{1,2, \ldots\}-$

$$
\begin{aligned}
C_{0}= & \sum_{n}\left(a_{n 0}^{-}+a_{n 0}^{+}\right) L_{n}(x) \\
& +\sum_{n m}\left(a_{n m}^{-}+a_{n m}^{+}\right) \zeta^{m} \frac{\sqrt{n+1+m}}{\sqrt{n+1}} L_{n}^{(m)}(x) \\
& +\sum_{n m}\left(b_{n m}^{-}+b_{n m}^{+}\right) \zeta^{* m} \frac{\sqrt{n+1+m}}{\sqrt{n+1}} L_{n}^{(m)}(x),(87) \\
C_{1}= & A+\sum_{n} A_{n} \zeta^{n+1}+B_{n} \zeta^{* n+1} \\
& +\sum_{n}\left(a_{n 0}^{-}-a_{n 0}^{+}\right) L_{n+1}(x) \\
& +\sum_{n m}\left(a_{n m}^{-}-a_{n m}^{+}\right) \zeta^{m} L_{n+1}^{(m)}(x) \\
& +\sum_{n m}\left(b_{n m}^{-}-b_{n m}^{+}\right) \zeta^{* m} L_{n+1}^{(m)}(x)
\end{aligned}
$$

$$
\begin{aligned}
C_{+}= & \sum_{n} \bar{A}_{n} \zeta^{n}+\frac{1}{2}\left\{\bar{a}_{n 0}^{-}+\bar{a}_{n 0}^{+}\right\} \zeta^{*} \frac{1}{\sqrt{n+1}} L_{n}^{(1)}(x) \\
& +\frac{1}{2} \sum_{n m}\left\{\bar{a}_{n m}^{-}-\bar{a}_{n m}^{+}\right\} \zeta^{m-1} \sqrt{n+1+m} L_{n+1}^{(m-1)}(x) \\
& +\frac{1}{2} \sum_{n m}\left\{\bar{b}_{n m}^{-}+\bar{b}_{n m}^{+}\right\} \zeta^{* m+1} \frac{1}{\sqrt{n+1}} L_{n}^{(m+1)}(x)
\end{aligned}
$$

in terms of generalized Laguerre polynomials $L_{n}^{(m)}(x)$ (cf. [17] Eq. (22.3.9)). The solution $C_{-}$can be inferred from $C_{+}$by replacing $\zeta \leftrightarrow \zeta^{*}, \bar{a}_{n m}^{ \pm} \leftrightarrow \bar{b}_{n m}^{ \pm}$and $\bar{A}_{n} \rightarrow \bar{B}_{n}$ and $\bar{a}_{n 0}^{-} \rightarrow-\bar{a}_{n 0}^{-}$.

\subsection{Special initial conditions}

\subsubsection{States of the atom}

If the atom was initially in the ground state, i.e. $\left\langle\sigma_{z}\right\rangle=-1$, and uncorrelated with the field, we have as initial conditions $C_{0}=0$ and $C_{ \pm}=0$, which results in vanishing values $\bar{A}_{n}(0)=\bar{B}_{n}(0)=\bar{a}_{n 0}^{ \pm}(0)=\bar{a}_{n m}^{ \pm}(0)=$ $\bar{b}_{n m}^{ \pm}(0)=0$ and in the properties $a_{n 0}^{+}(0)=-a_{n 0}^{-}$, $a_{n m}^{+}(0)=-a_{n m}^{-}(0)$ and $b_{n m}^{+}(0)=-b_{n m}^{-}(0)$. The remaining parameters have to be determined from the function $C_{1}$,

$$
\begin{aligned}
C_{1}=A & +\sum_{n} A_{n}(0) \zeta^{n+1}+B_{n}(0) \zeta^{* n+1}+2 a_{n 0}^{-} L_{n+1}(x) \\
& +2 \sum_{n m}\left\{a_{n m}^{-}(0) \zeta^{m}+b_{n m}^{-}(0) \zeta^{* m}\right\} L_{n+1}^{(m)}(x) .
\end{aligned}
$$

If the atom was excited in the beginning, i.e. $\left\langle\sigma_{z}\right\rangle=1$, and uncorrelated with the field, we have as initial conditions $C_{1}=0$ and $C_{ \pm}=0$ : again $\bar{A}_{n}(0)=\bar{B}_{n}(0)=$ $\bar{a}_{n 0}^{ \pm}(0)=\bar{a}_{n m}^{ \pm}(0)=\bar{b}_{n m}^{ \pm}(0)=0$ vanishing and now also $A=A_{n}(0)=B_{n}(0)=0$. But now we have $a_{n 0}^{+}(0)=a_{n 0}^{-}$, $a_{n m}^{+}(0)=a_{n m}^{-}(0)$ and $b_{n m}^{+}(0)=b_{n m}^{-}(0)$ and the remaining parameters have to be determined from the function $C_{0}$ :

$$
\begin{aligned}
C_{0} & =2 \sum_{n} a_{n 0}^{-} L_{n}(x) \\
& +2 \sum_{n m}\left\{a_{n m}^{-}(0) \zeta^{m}+b_{n m}^{-}(0) \zeta^{* m}\right\} \frac{\sqrt{n+1+m}}{\sqrt{n+1}} L_{n}^{(m)}(x) .
\end{aligned}
$$

Expansions in terms of generalized Laguerre polynomials are unambiguous, since these polynomials form a complete orthogonal system ([17] Eq. (22.2.12)).

\subsubsection{Field states}

The normally ordered characteristic function appears in number representation as (with $m=n^{\prime}-n$ )

$$
\begin{array}{r}
C\left(\xi, \xi^{*}\right)=\sum_{n \leq n^{\prime}} \frac{\xi^{m}\left\langle n|\varrho| n^{\prime}\right\rangle+\left(-\xi^{*}\right)^{m}\left\langle n^{\prime}|\varrho| n\right\rangle}{1+\delta_{n n^{\prime}}} \\
\times \sqrt{\frac{n !}{n^{\prime} !}} L_{n}^{(m)}\left(\xi \xi^{*}\right) .
\end{array}
$$


(Here we allow also for $m=0$.) For a thermal state the density matrix elements are given by

$$
\left\langle n|\varrho| n^{\prime}\right\rangle=\delta_{n n^{\prime}}(1-z) z^{n}
$$

with $z=\exp (-\hbar \omega / k T)<1$, whereas for a Glauber state with $\varrho=|\alpha\rangle\langle\alpha|$

$$
\left\langle n|\varrho| n^{\prime}\right\rangle=\exp \left(-|\alpha|^{2}\right) \alpha^{n} \alpha^{* n^{\prime}} / \sqrt{n ! n^{\prime} !} .
$$

A laser state of the field is a phase averaged Glauber state, i.e.

$$
\left\langle n|\varrho| n^{\prime}\right\rangle=\delta_{n n^{\prime}} \exp \left(-|\alpha|^{2}\right)|\alpha|^{2 n} / n ! .
$$

We thus have as characteristic function for a Glauber state

$$
\begin{aligned}
& C\left(\xi, \xi^{*}\right)=\exp \left(-|\alpha|^{2}\right) \\
& \quad \times \sum_{n m} \frac{|\alpha|^{2 n}}{(n+m) !} \frac{\left(\alpha^{*} \xi\right)^{m}+\left(-\alpha \xi^{*}\right)^{m}}{1+\delta_{m 0}} L_{n}^{(m)}\left(\xi \xi^{*}\right),
\end{aligned}
$$

whereas for a laser state there are only the terms with $m=0$.

\subsection{Number of field quanta}

The expectation value of the number of field quanta can now be calculated as

$$
\left\langle\Psi^{\dagger} \Psi\right\rangle=-\left.\frac{\partial^{2}\left(C_{0}+C_{1}\right)}{\partial \zeta \partial \zeta^{*}}\right|_{\zeta=\zeta^{*}=0} .
$$

Because

$$
\left.\frac{\partial^{2}\left(\zeta^{l} f(x)+\zeta^{* m} g(x)\right.}{\partial \zeta \partial \zeta^{*}}\right|_{\zeta=\zeta^{*}=0}=\delta_{l 0} f^{\prime}(0)+\delta_{m 0} g^{\prime}(0)
$$

and $L_{n}^{(m) '}(0)=-\left(\begin{array}{c}n+m \\ n-1\end{array}\right)$ we get

$$
\left\langle\Psi^{\dagger} \Psi\right\rangle=\sum_{n}(2 n+1) a_{n 0}^{-}-a_{n 0}^{+}(t) .
$$

This can be interpreted physically as follows: if the atom was initially excited (upper case) or in the ground state (lower case), we have

$$
\left\langle\Psi^{\dagger} \Psi\right\rangle=\sum_{n}\left\{2 n+1 \mp \cos \left(\Omega_{n} t\right)\right\} a_{n}
$$

with

$$
a_{n}=\left\{\begin{array}{l}
\frac{1}{2} \varrho_{n}(0) \\
\frac{1}{2} \varrho_{n+1}(0)
\end{array},\right.
$$

since $a_{n}$ depends on the initial probability for $n$ resp. $n+1$ photons. If the field began in a Glauber state $|\alpha\rangle$ (i.e. with $\left.\varrho_{n}=\exp \left(-|\alpha|^{2}\right)|\alpha|^{2 n} / n !\right)$ with $\exp \left(-|\alpha|^{2}\right) \ll 1$ we get in accordance with equation (51)

$$
\sum_{n}(2 n+1) a_{n} \approx|\alpha|^{2} \pm \frac{1}{2}
$$

whereas the sum over $a_{n} \cos \left(\Omega_{n} t\right)$ leads for $|\alpha| \gg 1$ to the collapse and revival already mentioned in Figure 7. Many terms seem to contribute here. However, $x^{n} / n$ ! is particularly large at $n \approx x-\frac{1}{2}$. Therefore we expand $\Omega_{n}$ around $n \approx|\alpha|^{2} \pm \frac{1}{2}$ and take only the first two terms. In terms of $\alpha_{ \pm}=\sqrt{|\alpha|^{2} \pm \frac{1}{2}}$ the result reads

$$
\begin{aligned}
& -\sum_{n} a_{n 0}^{+}(t)=\mp \frac{1}{2} \exp \left(-|\alpha|^{2}+|\alpha|^{2} \cos \frac{g t}{2 \alpha_{ \pm}}\right) \\
& \times \cos \left(\frac{\alpha_{ \pm} g t}{2}+|\alpha|^{2} \sin \frac{g t}{2 \alpha_{ \pm}}+\frac{1 \pm 1}{2} \frac{g t}{2 \alpha_{ \pm}}\right) .
\end{aligned}
$$

If the atom was initially in the ground state (lower sign), the result for small $t$ and large $|\alpha|$ is already known elsewhere [24] in the form

$$
\left\langle\Psi^{\dagger} \Psi\right\rangle \approx|\alpha|^{2}-\frac{1}{2}+\frac{1}{2} \exp \left\{-\frac{1}{8}(g t)^{2}\right\} \cos |\alpha g t| .
$$

According to this the amplitude does not depend on the intensity $|\alpha|^{2}$ and is called collapse function [25]. Our expression in equation (103) even shows the revival (Fig. 8), however not with the correct amplitude - one should also have included higher terms. Comparison with Figure 5 shows, that the parameters used [9] do not allow for a revival because the damping is not small enough.

\section{Damped solutions}

The solutions including damping are more complicated as they require generalized hypergeometric functions. We do not give these "solutions", because there are no orthogonality relations known even for normal hypergeometric functions. Thus these functions can not be used to fit the initial distributions.

We propose to use solutions, that take advantage of $\left(\gamma_{\mathrm{L}}, \gamma_{\mathrm{P}}, \gamma_{\mathrm{A}}\right) \ll g$. We already know the time dependent factors and fit the initial values to the unperturbed terms using the old equations (87-89); the effect of damping is incorporated only in the time dependence of the auxiliary functions,

$$
\begin{aligned}
a_{n m}^{ \pm}(t)= & a_{n m}^{ \pm}(0) \exp \left(-\gamma_{n+m, n} t\right) \cos \left(\Omega_{n+m, n}^{ \pm} t\right) \\
& +\bar{a}_{n m}^{ \pm}(0) \exp \left(-\gamma_{n+m, n} t\right) \sin \left(\Omega_{n+m, n}^{ \pm} t\right) \\
\bar{a}_{n m}^{ \pm}(t)= & \bar{a}_{n m}^{ \pm}(0) \exp \left(-\gamma_{n+m, n} t\right) \cos \left(\Omega_{n+m, n}^{ \pm} t\right) \\
& -a_{n m}^{ \pm}(0) \exp \left(-\gamma_{n+m, n} t\right) \sin \left(\Omega_{n+m, n}^{ \pm} t\right),
\end{aligned}
$$

and $b_{n m}^{ \pm}(t)$ and $\bar{b}_{n m}^{ \pm}(t)$ of the same form. Here the parameters

$$
\gamma_{n}=(2 n+1) \gamma_{\mathrm{L}}+\gamma_{\mathrm{A}}+\gamma_{\mathrm{P}}
$$

and

$$
\gamma_{n n^{\prime}}=\frac{1}{2}\left(\gamma_{n}+\gamma_{n^{\prime}}\right)
$$




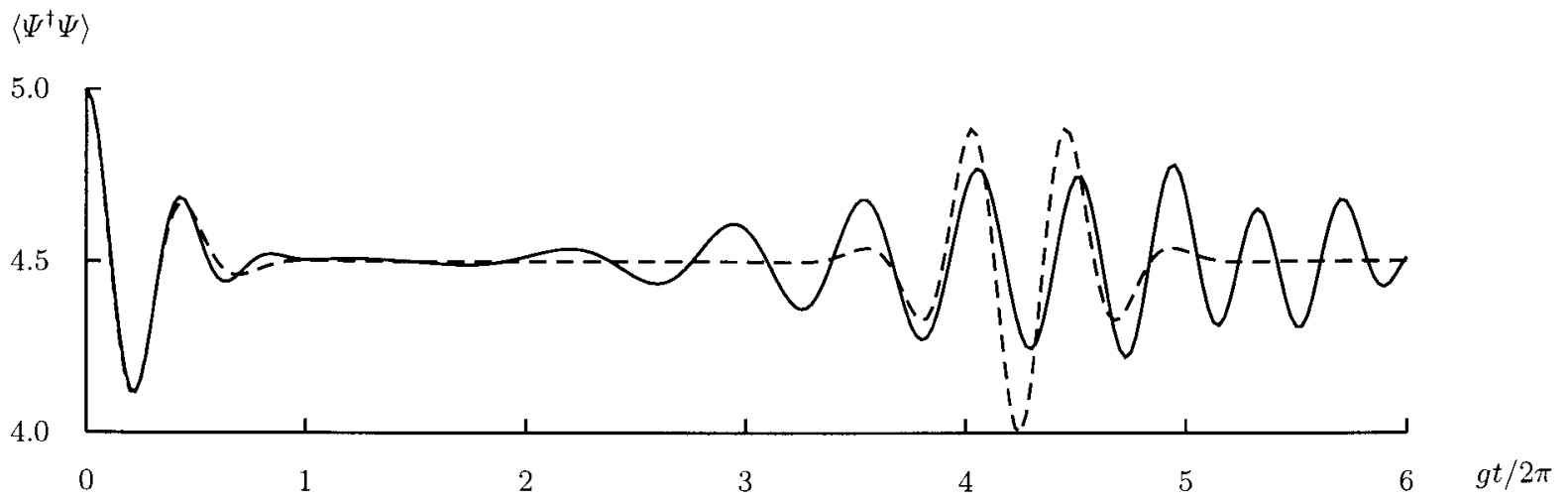

Fig. 8. Expectation value of the photon number as function of time without damping, if there was initially a Glauber state with 5 photons and the atom in the ground state: (-) exact calculation, (- - -) with the approximation equation (103).

i.e.

$$
\gamma_{n+m, n}=\gamma_{\mathrm{L}}+\gamma_{\mathrm{A}}+\gamma_{\mathrm{P}}+(2 n+m) \gamma_{\mathrm{L}}
$$

are defined similarly to $\Omega_{n n^{\prime}}^{+}$in equation (15).

Furthermore we employ

$$
\begin{aligned}
a_{n 0}^{+}(t)= & a_{n 0}^{+}(0) \exp \left(-\gamma_{n} t\right) \cos \left(\Omega_{n} t\right) \\
& \left.+\bar{a}_{n 0}^{+}(0) \exp \left(-\gamma_{n} t\right) \sin \left(\Omega_{n} t\right)\right\} \\
\bar{a}_{n 0}^{+}(t)= & \bar{a}_{n 0}^{+}(0) \exp \left(-\gamma_{n} t\right) \cos \left(\Omega_{n} t\right) \\
& -a_{n 0}^{+}(0) \exp \left(-\gamma_{n} t\right) \sin \left(\Omega_{n} t\right)
\end{aligned}
$$

The terms with $a_{n 0}^{-}$are already known, namely equal to $\frac{1}{4} \sum_{\varepsilon}\langle\varepsilon, n|\varrho| \varepsilon, n\rangle$. This quantity does not change in time if there is no damping - and we have determined the sum in equation (31) resp. equation (43).

The terms with $\bar{a}_{n 0}^{-}$could be applied without any Laguerre polynomial, since we could work with any differentiable function $f$ :

$$
C_{+}=\frac{1}{2} \zeta^{*} \exp \left\{-\left(\gamma_{0}+\gamma_{\mathrm{P}}\right) t\right\} f\left(x \mathrm{e}^{-2 \gamma_{\mathrm{L}} t}\right)
$$

and

$$
C_{-}=-\frac{1}{2} \zeta \exp \left\{-\left(\gamma_{0}+\gamma_{\mathrm{P}}\right) t\right\} f\left(x \mathrm{e}^{-2 \gamma_{\mathrm{L}} t}\right)
$$

are exact solutions, yet we may also use

$$
\bar{a}_{n 0}^{-}(t)=\exp \left\{-\left(\gamma_{n}+\gamma_{\mathrm{P}}\right) t\right) \bar{a}_{n 0}^{-}(0) .
$$

We have the pair

$$
\begin{aligned}
& A_{n}(t)=A_{n}(0) \exp \left(-\frac{1}{2} \gamma_{n} t\right) \cos \left(\frac{1}{2} \Omega_{n} t\right) \\
&+\left(g \bar{A}_{n}(0)-\frac{\gamma_{\mathrm{L}}-\gamma_{\mathrm{A}}}{2} A_{n}(0)\right) \\
& \times \exp \left(-\frac{1}{2} \gamma_{n} t\right) \frac{\sin \left(\frac{1}{2} \Omega_{n} t\right)}{\frac{1}{2} \Omega_{n}}, \\
& \bar{A}_{n}(t)=\bar{A}_{n}(0) \exp \left(-\frac{1}{2} \gamma_{n} t\right) \cos \left(\frac{1}{2} \Omega_{n} t\right) \\
&-\left(\frac{\left(\frac{1}{2} \Omega_{n}\right)^{2}}{g} A_{n}(0)-\frac{\gamma_{\mathrm{L}}-\gamma_{\mathrm{A}}}{2} \bar{A}_{n}(0)\right) \\
& \times \exp \left(-\frac{1}{2} \gamma_{n} t\right) \frac{\sin \left(\frac{1}{2} \Omega_{n} t\right)}{\frac{1}{2} \Omega_{n}},
\end{aligned}
$$

and $B_{n}(t)$ and $\bar{B}_{n}(t)$ of the same form and a constant $A$.

It follows from equations $(10,26)$, that this yields the correct time dependent basis.

\subsection{Number of field quanta}

When beginning with a Glauber state, one part of the expression equation (99) is already known from equation (51). Still missing is the term

$$
\begin{aligned}
-\sum_{n} a_{n 0}^{+}(t)= & \mp \frac{1}{2} \exp \left[-|\alpha|^{2}-\left(\gamma_{\mathrm{L}}+\gamma_{\mathrm{A}}+\gamma_{\mathrm{P}}\right) t\right] \\
& \times|\alpha|^{1 \mp 1} \sum_{n} \frac{\tau^{n}}{\left(n+\frac{1}{2} \mp \frac{1}{2}\right) !} \cos \Omega_{n} t .
\end{aligned}
$$

This can be approximated by the same procedure as that leading to equation (103) and results in

$$
\begin{aligned}
&-\sum_{n} a_{n 0}^{+}(t) \approx \mp \frac{1}{2} \exp \left(-|\alpha|^{2}-\left(\gamma_{\mathrm{A}}+\gamma_{\mathrm{P}} \pm \gamma_{\mathrm{L}}\right) t+\tau \cos \frac{g t}{2 \tau_{ \pm}}\right) \\
& \times \cos \left(\frac{\tau_{ \pm} g t}{2}+\tau \sin \frac{g t}{2 \tau_{ \pm}}+\frac{1 \pm 1}{2} \frac{g t}{2 \tau_{ \pm}}\right),
\end{aligned}
$$

if we - stressing the generalization against equation (103) - use $\tau_{ \pm}=\sqrt{\tau \pm \frac{1}{2}}$, where $\tau$ is given in equation (42). As before, the upper sign belongs to an atom initially excited and the lower sign to an atom initially in the ground state.

Figure 9 shows an example.

\section{Summary and outlook}

Since it is impossible to completely decouple real physical systems from their macroscopic environment they should be treated as open. We took the Jaynes-Cummings model allowing for damping with the time dependence given by the Liouville (master) equation (5) which results in a non unitary dynamics and causes irreversibilty [26]. Observables begin their evolution linearly in time, not quadratically $-i$.e. like $\exp (-\gamma t) \cos \omega t$ instead of $\cos \omega t$. This is 


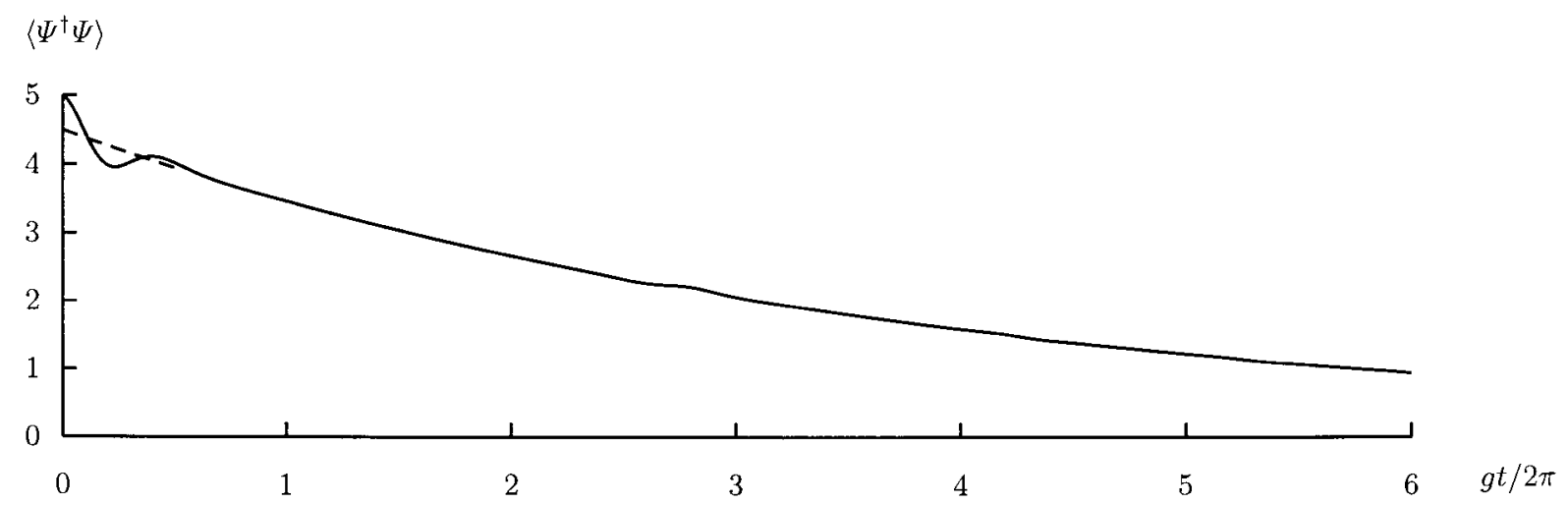

Fig. 9. Change of Figure 8 due to damping: expectation value of the number of photons as a function of time, if initially there was a Glauber state with 5 photons and an atom in the ground state, after equations $(51,116)$. In comparison to a Fock state (cf. Fig. 5) there are less oscillations for a Glauber state, because in this case the contributions of the constituent Fock states average out after a short time (collapse). The broken line displays the contribution of equation (51).

an important aspect with regard to the quantum Zeno effect $[27,28]$ where a true stopping of the dynamics in the limit of frequently repeated measurements relies on the quadratic time dependence. With a residual linear contribution the dynamics cannot be stopped but at most slowed down. The linearity for small $t$ is also an important ingredient for deriving Fermi's golden rule. As shown in one of Döring's textbooks [29] this rule is often deduced from incorrect arguments. The rule follows however for open systems [8].

The differential equation was solved in the basis of dressed states (Eq. (2)) diagonalizing the JaynesCummings Hamiltonian. (The master equation may also be integrated numerically [22] resulting in the same numbers as given here.) Our procedure is motivated by the fact that the Hamiltonian contains large frequencies and the damping is described by smaller constants.

The main effect of the transition from a closed to an open system is the rather fast "decoherence" [30]: the collapse of the wave function is a consequence rather than an additional postulate [26]. The collapse of the non diagonal density matrix elements in energy representation is displayed clearly in Figures 1 to 3 . It should be kept in mind that without damping the curves in the upper part of Figure 1 would be horizontal lines and the rest of Figures 1 to 3 would show oscillations without damping.

The system of a two-level atom (described by a $2 \times 2$ density matrix) coupled to a harmonic oscillator is described by four density matrices of harmonic oscillators. Since we normally are interested only in a few observables we have discussed not only the time dependence of the density operator but also the time dependence of observables. Here it is important to pay regard to the correlations between the degrees of freedom - erroneously neglected in the quasi classical approximation. This can be handled with a system of partial differential equations for the characteristic functions. Damping, however, leads to generalized hypergeometric functions which can not be fitted to the initial values exactly but only approximately.
This has been shown for the number of field quanta as an example.

We thank Dr. Christof Wunderlich at our institute for valuable suggestions. This work was supported by Deutsche Forschungsgemeinschaft SSP Zeitabhängige Phänomene und Methoden in Quantensystemen der Physik und Chemie.

\section{References}

1. E.T. Jaynes, F.W. Cummings, Proc. IEEE 51, 89 (1963).

2. P. Meystre, M. Sargent III, Elements of Quantum Optics (Springer, Berlin, 1991).

3. D.F. Walls, G.J. Milburn, Quantum Optics (Springer, Berlin, 1994).

4. S. Haroche, Rydberg atoms and radiation in a resonant cavity, in Tendances actuelles en physique atomique (Les Houches, 1982), edited by G. Grynberg, R. Stora (NorthHolland, Amsterdam, 1982), pp. 193-309.

5. F. Haake, Statistical Treatment of Open Systems by Generalized Master Equations, in Quantum Statistics in $O p$ tics and Solid State Physics (Springer Tracts in Modern Physics, Vol. 66, 1973), edited by G. Höhler (Springer, Berlin, 1973), pp. 98-168.

6. G. Lindblad, Commun. Math. Phys. 48, 119 (1976).

7. U. Weiss, Quantum Dissipative Systems, Series in Modern Condensed Matter Physics (World Scientific, Singapore, 1993), Vol. 2.

8. A. Lindner, Grundkurs Theoretische Physik (2. Auflage) (Teubner, Stutgart, 1997).

9. M. Brune, E. Hagley, J. Dreyer, X. Maître, A. Maali, C. Wunderlich, J.-M. Raimond, S. Haroche, Phys. Rev. Lett. 77, 4887 (1996).

10. P. Münstermann, T. Fischer, P. Maunz, P.W.H. Pinkse, G. Rempe, Phys. Rev. Lett. 82, 3791 (1999).

11. J. Ye, D.W. Vernooy, H.J. Kimble, Phys. Rev. Lett. 83, 4987 (1999).

12. J. Eiselt, H. Risken, Opt. Commun. 72, 351 (1989).

13. B. Daeubler, H. Risken, L. Schoendorff, Phys. Rev. A 48, 3955 (1993).

14. A.J.V. Wonderen, Phys. Rev. A 56, 3116 (1997). 
15. H.-J. Briegel, B.G. Englert, Phys. Rev. A 47, 3311 (1993).

16. J.G. Peixoto de Farina, M.C. Nemes, Phys. Rev. A 59, 3918 (1999).

17. M. Abramowitz, I.A. Stegun, Handbook of Mathematical Functions (Dover, New York, 1972).

18. P. Meystre, A. Quattropani, H.P. Baltes, Phys. Lett. A 49 , 85 (1974).

19. R. Zimmermann, J. Wauer, J. Lumin. 58, 271 (1994).

20. J. Wauer, Elektron-Phonon-Relaxation mit Gedächtnis, Dissertation an der HU, Berlin, 1996.

21. A. Lindner, G. Quehl, Jaynes-Cummings-Modell mit Dämpfung, in DPG-Frühjahrstagung (Mainz, 1997), Verhandl.d.DPG (VI)32, p. 344.

22. G. Quehl, Über die Zeitabhängigkeit von Beobachtungsgrößen beim Zwei-Niveau-System, Harmonischen Oszillator und Jaynes-Cummings-Modell, Diplomarbeit,
Hamburg, 1997.

23. R. Zimmermann, J. Wauer, A. Leitenstorfer, C. Fürst, J. Lumin. 76\&77, 34 (1998).

24. P. Meystre, E. Geneux, A. Quattropani, A. Faist, Nuovo Cimento B 25, 521 (1975).

25. J.H. Eberly, N.B. Narozhny, J.J. Sanchez-Mondragon, Phys. Rev. Lett. 44, 1323 (1980).

26. N.G. van Kampen, Physica A 153, 97 (1988).

27. B. Misra, E.C.G. Sudarshan, J. Math. Phys. 18, 756 (1977).

28. V. Frerichs, A. Schenzle, Phys. Rev. A 44, 1962 (1991).

29. W. Döring, Atomphysik und Quantenmechanik 3. Anwendungen (de Gruyter, Berlin, 1979).

30. D. Giulini, E. Joos, C. Kiefer, J. Kupsch, I.-O. Stamatescu, H.D. Zeh, Decoherence and the Appearance of a Classical World in Quantum Theory (Springer, Berlin, 1996). 